\title{
The Angular Momentum Evolution of Very Low Mass Stars
}

\author{
Alison Sills, M. H. Pinsonneault, D. M. Terndrup \\ Department of Astronomy, The Ohio State University, 174 W. 18th Ave., Columbus, OH, \\ 43210, USA
}

\begin{abstract}
We present theoretical models of the angular momentum evolution of very low mass stars $\left(0.1-0.5 \mathrm{M}_{\odot}\right)$. We also present models of solar analogues $(0.6$ $1.1 \mathrm{M}_{\odot}$ ) for comparison with previous work. We investigate the effect of rotation on the effective temperature and luminosity of these stars. Rotation lowers the effective temperature and luminosity of the models relative to standard models of the same mass and composition. We find that the decrease in $\mathrm{T}_{\text {eff }}$ and $\mathrm{L}$ can be significant at the higher end of our mass range, but becomes small below $0.4 \mathrm{M}_{\odot}$. The effects of different assumptions about internal angular momentum transport are discussed. Formulae for relating $\mathrm{T}_{\text {eff }}$ to mass and $\mathrm{v}_{\text {rot }}$ are presented. We demonstrate that the kinetic energy of rotation is not a significant contribution to the luminosity of low mass stars.

Previous studies of the angular momentum evolution of low mass stars concentrated on solar analogues and were complicated by uncertainties related to the internal transport of angular momentum. In this paper we extend our theoretical models for the angular momentum evolution of stars down to 0.1 $\mathrm{M}_{\odot}$. We compare our models to rotational data from young open clusters of different ages to infer the rotational history of low mass stars, and the dependence of initial conditions and rotational evolution on mass. We find that the qualitative conclusions for stars below $0.6 \mathrm{M}_{\odot}$ do not depend on the assumptions about internal angular momentum transport with the exception of a zero-point shift in the angular momentum loss saturation threshold. We argue that this makes these low mass stars ideal candidates for the study of the angular momentum loss law and distribution of initial conditions. For stars with masses between 0.6 and $1.1 \mathrm{M}_{\odot}$, scaling the saturation threshold by the Rossby number can reproduce the observed mass dependence of the stellar angular momentum evolution. We find that neither models with solid body rotation nor differentially rotating models can simultaneously reproduce the observed stellar spin down in the 0.6 to $1.1 \mathrm{M}_{\odot}$ mass range and for stars between 0.1 and 0.6 $\mathrm{M}_{\odot}$. We argue that the most likely explanation is that the saturation threshold drops more steeply at low masses than would be predicted with a simple Rossby
\end{abstract}


scaling. In young clusters there is a systematic increase in the mean rotation rate with decreased temperature below $3500 \mathrm{~K}\left(0.4 \mathrm{M}_{\odot}\right)$. This suggests either inefficient angular momentum loss or mass-dependent initial conditions for stars near the fully convective boundary.

Subject headings: stars: evolution - stars: rotation - stars: interiors - stars: formation - low mass stars

\section{Introduction}

The study of stellar rotation can provide insights into a variety of interesting subjects in the fields of star formation and stellar structure and evolution. The observed rotation velocities and rotation periods of open cluster stars as a function of mass and age yield clues about the star formation process, the internal transport of angular momentum, the loss of angular momentum through magnetized stellar winds, and the origin and generation of stellar magnetic fields. In addition, rotation can drive mixing not present in standard stellar models with important consequences for the observed surface abundances of stars.

We are now able to observe significant samples of stars down to the hydrogen burning limit in open clusters (e.g. NGC 2420, von Hippel et al. 1996), globular clusters (e.g. 47 Tucanae, Santiago, Elson \& Gilmore 1996), and the field (e.g. Tinney, Mould \& Reid 1993). We have also been able to observe rotation in these stars, using both spectroscopy to determine $v \sin i$ (Kraft 1965, Stauffer et al. 1997a), and photometry to monitor spot modulation on the stars (Barnes et al. 1999, Prosser et al. 1995) and thereby determine rotational periods. This plethora of information about the rotation of low mass stars has been a great boon to the study of these stars for a number of reasons. First of all, the rotation rates of stars on the main sequence are determined by their pre-main sequence evolution, so that by studying the rotational evolution of low mass stars, we can investigate the early stages of stellar evolution. Second, stellar magnetic phenomena are related to stellar rotation. The evolution of the rotation rates is largely determined by angular momentum loss from a magnetized stellar wind (Kawaler 1988, Weber \& Davis 1967). Stellar rotation is found to correlate with chromospheric activity and other magnetic tracers (for a review see Hartmann \& Noyes 1987), which lends support to the idea that rotation plays a crucial role in the generation of stellar magnetic fields, through the operation of a dynamo.

In recent years there have been a large number of observational and theoretical studies

of the angular momentum evolution of low mass stars (see Krishnamurthi et al. 1997 for 
a review). These studies have focused on solar analogues which have the most extensive observational database. However, the majority of the moment of inertia of solar analogues is in the radiative interior, so theoretical predictions for their angular momentum evolution are strongly influenced by the treatment of internal angular momentum transport. In this paper we examine the rotational properties of stellar models of lower mass stars where the radiative core provides a smaller (or even nonexistent) fraction of the moment of inertia. We will show that the study of these low mass stars can provide valuable clues about angular momentum loss and the distribution of initial conditions that depend only weakly on the treatment of internal angular momentum transport. We begin with a discussion of the important ingredients for theoretical models of stellar angular momentum evolution.

The rotational evolution of a low mass star is determined by four factors. The first factor is the star's initial angular momentum. Young low mass stars are fully convective, and helioseismic data indicate that the rotation rate in the solar convective zone is independent of radius; as a result, the initial angular momentum is fully specified by the initial rotation period. Observed T Tauri star rotation periods are between 2 and 16 days, with an average of 9.54 days and a median of 8.5 days (Choi \& Herbst 1996).

The rate at which angular momentum is lost through a magnetic wind will also strongly influence the stellar rotation rate. In a linear dynamo the angular momentum loss rate is proportional to the surface angular velocity cubed (Weber \& Davis 1967, Kawaler 1988). However, an angular momentum loss law of this form predicts rapid spin down of fast rotators in disagreement with observational data (Pinsonneault et al. 1990). More recent theoretical models use an angular momentum loss law that saturates at some critical rotation rate; the lower the saturation threshold, the longer that rapid rotation can persist. Previous work on solar analogues (Barnes \& Sofia 1996, Krishnamurthi et al. 1997) has suggested that the saturation threshold depends on mass, and is inversely proportional to the global convective overturn timescale. This scaling is prompted by its relationship to the Rossby number, a measure of the strength of dynamo magnetic activity in the star, and supported by observational data on the relationship between chromospheric and coronal activity indicators, rotation, and mass (e.g. Noyes et al. 1984, Patten \& Simon 1996, Krishnamurthi et al. 1998).

The rotation history of a star is also influenced by the internal transport of angular momentum. If a torque is applied to the surface convection zone a shear will be generated between the convective envelope and radiative core; the angular momentum evolution will depend on the efficiency of the angular momentum coupling between the core and envelope.

One straightforward model is to assume that the coupling time scale is extremely short, i.e. that solid body rotation is enforced at all times throughout the star. This is predicted 
in the limiting case of strong magnetic coupling between the core and envelope (see for example Charbonneau \& MacGregor 1993), and models of this type have been investigated in the context of the angular momentum evolution of solar analogues (Collier-Cameron \& Jianke 1994; Bouvier, Forestini, \& Allain 1997; Krishnamurthi et al. 1997).

Another approach is to solve for the internal transport of angular momentum by hydrodynamic mechanisms (see Pinsonneault 1997 for a review), internal gravity waves (Talon \& Zahn 1998), magnetic fields (Charbonneau \& MacGregor 1993), or hybrid models including more than one of these mechanisms (e.g. Barnes, Charbonneau, \& MacGregor 1999). In these cases, angular momentum can be stored in a rapidly rotating core. The rotational evolution of models of this class can therefore be different than that of solid body models as that reservoir of angular momentum can be shielded from the strong angular momentum loss that accompanies rapid surface rotation.

One of the most challenging features of the open cluster data for theoretical models to explain is the large number of young slowly rotating stars. A simple projection of T Tauri star rotation velocities with conservation of angular momentum would predict more rapid rotation than is observed, and the short time scale would not permit sufficient angular momentum loss given the modest predicted rotation rates (see for example Stauffer \& Hartmann 1987, Keppens, MacGregor, \& Charbonneau 1995). Königl (1991) proposed that the presence of an accretion disk, coupled to a protostar by a magnetic field, will force the protostar to rotate with the same period as the disk (see also Keppens, MacGregor, \& Charbonneau 1995; Collier-Cameron, Campbell, \& Quaintrell 1995). Only when the disk is disrupted can the star begin its normal angular momentum evolution (spin-up because of contraction modified by the processes described above). When the star is no longer locked to the disk, it begins to spin up as its radius contracts. Since the star is starting with a smaller moment of inertia, it can never spin up as much as a star which lost its disk at the birthline. This would produce a range of surface rotation rates from a range of accretion disk lifetimes and provided an attractive physical explanation for the observed distribution of stellar surface rotation rates. There is indirect evidence for the model (Edwards et al. 1993, Choi \& Herbst 1996), in the sense that the distribution of rotation periods is different for young stars with infrared excesses than for young stars without them (but see also Stassun et al. 1999). The longest reasonable disk lifetime, as determined from studies of T Tauri stars, is about $10 \mathrm{Myr}$ (Strom et al. 1989). We therefore wish to reproduce the slowest rotators in each cluster with models which have disk lifetimes of 10 Myr or less. This creates problems for solid body models, since they require much longer disk lifetimes and also have difficulty in explaining the observed spindown of slow rotators on the main sequence (Krishnamurthi et al. 1997, Allain 1998). 
The ingredients which determine the rotational evolution of low mass stars are not independent. For example, a rapidly rotating star could exist because it had a short initial period. It could be rotating rapidly because it has not lost much angular momentum since the wind saturation threshold is low. Or, it could have been locked to its disk for a very short period of time. However, this degeneracy of factors is not an insoluble problem. We can determine the angular momentum loss rate (the saturation threshold) by comparing rotation rates of the fastest rotators in clusters of different ages. These stars must have had the fastest initial periods and have not been locked to disks. Therefore, any change in their rotation rates will be caused by their contraction to the main sequence, plus any loss due to their wind. We have evidence from solar analogues that the magnetic wind saturation threshold depends on mass (Barnes \& Sofia 1996, Krishnamurthi et al. 1997). By modeling the fastest rotators in a series of clusters with different ages, we can constrain this threshold for each mass. After we know the dependence of the saturation threshold on mass, we can attempt to model the slowest rotators in young open clusters by allowing them to retain their disks for long periods of time.

By studying the lowest mass stars, we can simplify the problem. Stars with masses less than $\sim 0.5 \mathrm{M}_{\odot}$ are fully convective throughout their pre-main sequence evolution, while stars with masses less than $0.25 \mathrm{M}_{\odot}$ are fully convective for their entire lifetime. Helioseismic data suggest that they will therefore always rotate as solid bodies, so the internal transport of angular momentum in such stars can be modeled very simply. We can use fully convective stars to diagnose the initial conditions in open clusters, such as the range of disk lifetimes and initial rotation periods, and the mass dependence of the angular momentum loss law.

As discussed above, a number of researchers have investigated the angular momentum evolution of solar analogues $\left(0.8-1.2 \mathrm{M}_{\odot}\right)$. The major obstacles which prevented us from modeling very low mass stars accurately in the past have been the lack of adequate model atmospheres, opacities and equations of state for low temperatures (less than $4000 \mathrm{~K}$ ). Lately, several groups (Alexander \& Ferguson 1994; Allard \& Hauschildt 1995; Saumon, Chabrier \& Van Horn 1995) have made breakthroughs in the necessary physics. Improved evolutionary models of very low mass stars have been produced over the last few years (Baraffe et al. 1998). However, the effects of rotation have not been included in these recent low mass models, and neglecting rotation could lead to anomalous results. For example, rotation can modify the amount of lithium depletion in low mass stars, affecting the derived ages from lithium isochrone fitting (e.g. Stauffer, Schultz \& Kirkpatrick 1998). In this work, we present the first rotational models of stars less massive than $0.5 \mathrm{M}_{\odot}$. We also include models for stars up to $1.1 \mathrm{M}_{\odot}$, for comparison with previous work. In section 2 , we discuss the methods used to determine the rotational evolution of the low mass stars. 
We present the results in section 3, and discuss their implications in section 4 .

\section{Method}

We used the Yale Rotating Stellar Evolution Code (YREC) to construct models of the low mass stars. YREC is a Henyey code which solves the equations of stellar structure in one dimension (Guenther et al. 1992). The star is treated as a set of nested, rotationally deformed shells. The chemical composition of each shell is updated separately using the nuclear reaction rates of Gruzinov \& Bahcall (1998). The initial chemical mixture is the solar mixture of Grevesse \& Noels (1993), and our models have a metallicity of $\mathrm{Z}=0.0188$. Gravitational settling of helium and heavy elements is not included in these models. We use the latest OPAL opacities (Iglesias \& Rogers 1996) for the interior of the star down to temperatures of $\log T(K)=4$. For lower temperatures, we use the molecular opacities of Alexander \& Ferguson (1994). For regions of the star which are hotter than $\log T(K) \geq 6$, we used the OPAL equation of state (Rogers, Swenson \& Iglesias 1996). For regions where $\log T(K) \leq 5.5$, we used the equation of state from Saumon, Chabrier \& Van Horn (1995), which calculates particle densities for hydrogen and helium including partial dissociation and ionization by both pressure and temperature. In the transition region between these two temperatures, both formulations are weighted with a ramp function and averaged. The equation of state includes both radiation pressure and electron degeneracy pressure. For the surface boundary condition, we used the stellar atmosphere models of Allard \& Hauschildt (1995), which include molecular effects and are therefore relevant for low mass stars. We used the standard Böhm-Vitense mixing length theory (Cox \& Guili 1968; Böhm-Vitense 1958 ) with $\alpha=1.72$. This value of $\alpha$, as well as the solar helium abundance, $Y_{\odot}=0.273$, was obtained by calibrating models against observations of the solar radius $\left(6.9598 \times 10^{10} \mathrm{~cm}\right)$

and luminosity $\left(3.8515 \times 10^{33} \mathrm{erg} / \mathrm{s}\right)$ at the present age of the Sun $(4.57 \mathrm{Gyr})$.

The structural effects of rotation are treated using the scheme derived by Kippenhahn \& Thomas (1970) and modified by Endal \& Sofia (1976). The details of this particular implementation are discussed in Pinsonneault et al. (1989). In summary, quantities are evaluated on equipotential surfaces rather than the spherical surfaces usually used in stellar models. The mass continuity equation is not altered by rotation:

$$
\frac{\partial M}{\partial r}=4 \pi r^{2} \rho
$$

The equation of hydrostatic equilibrium includes a term which takes into account the modified gravitational potential of the non-spherical equipotential surface:

$$
\frac{\partial P}{\partial M}=-\frac{G M}{4 \pi r^{4}} f_{P}
$$


where

$$
f_{P}=\frac{4 \pi r^{4}}{G M S} \frac{1}{\left\langle g^{-1}\right\rangle},
$$

and

$$
\left\langle g^{-1}\right\rangle=\frac{1}{S} \int_{\psi=\text { const }} g^{-1} d \sigma,
$$

$S$ is the surface area of an equipotential surface, and $d \sigma$ is an element of that surface. The factor $f_{P}$ is less than one for non-zero rotation, and approaches one as the rotation rate goes to zero. The radiative temperature gradient also depends on rotation:

$$
\frac{\partial \ln T}{\partial \ln P}=\frac{3 \kappa}{16 \pi a c G} \frac{P}{T^{4}} \frac{L}{M} \frac{f_{T}}{f_{P}},
$$

where

$$
f_{T}=\left(\frac{4 \pi r^{2}}{S}\right)^{2} \frac{1}{\langle g\rangle\left\langle g^{-1}\right\rangle},
$$

and $\langle g\rangle$ is analogous to $\left\langle g^{-1}\right\rangle . f_{T}$ has the same asymptotic behaviour as $f_{P}$, but is typically much closer to 1.0. The energy conservation equation retains its non-rotating form. Therefore, all the structural effects of rotation are limited to the equation of hydrostatic equilibrium and the radiative temperature gradient. This modified temperature gradient is used in the Schwarzschild criterion for convection:

$$
\frac{\partial \ln T}{\partial \ln P}=\min \left[\nabla_{a d}, \nabla_{r a d} \frac{f_{T}}{f_{P}}\right]
$$

where $\nabla_{a d}$ and $\nabla_{r a d}$ are the normal spherical adiabatic and radiative temperature gradients.

The Endal \& Sofia scheme is valid across a wide range of rotation rates, for a restricted class of angular momentum distributions. This scheme requires that the rotational velocity is constant on equipotential surfaces, which does not allow for modeling of latitude-dependent rotational profiles. We assume that horizontal turbulence is sufficiently strong to enforce spherical rotation (Chaboyer \& Zahn 1992). The other restriction on the Endal \& Sofia scheme is that it assumes that the potential is conservative, which is not valid when the star is expanding or contracting. Therefore, it is necessary to take small timesteps during any phase of expansion or contraction (such as the pre-main sequence) to minimize the errors introduced by this limitation. This method, and most others used in rotational stellar evolution codes, does not include the horizontal transport of heat, which may be important in the most rapidly rotating stars, rotating very close to their breakup velocities. See Meynet \& Maeder (1997) for a detailed discussion of the validity of this approach to the evolution of rotating stars. 
To model the loss of angular momentum from the surface, we adopt a modified Kawaler angular momentum loss rate with a $\mathrm{N}=1.5$ wind law (Chaboyer, Demarque \& Pinsonneault 1995), given by

$$
\begin{gathered}
\frac{d J}{d t}=-K \omega^{3}\left(\frac{R}{R_{\odot}}\right)^{0.5}\left(\frac{M}{M_{\odot}}\right)^{-0.5}, \omega \leq \omega_{\text {crit }} \\
\frac{d J}{d t}=-K \omega_{c r i t}^{2} \omega\left(\frac{R}{R_{\odot}}\right)^{0.5}\left(\frac{M}{M_{\odot}}\right)^{-0.5}, \omega>\omega_{\text {crit }}
\end{gathered}
$$

This represents the draining of angular momentum from the outer convection zone through a magnetic wind. The magnetic field is assumed to be proportional to the angular velocity while that velocity is small, but then saturates at $\omega=\omega_{\text {crit }}$. The constant $K$ is calibrated by reproducing the solar rotation rate $(2 \mathrm{~km} / \mathrm{s})$ at the solar age (4.57 Gyr from the birthline) for a $1.0 \mathrm{M}_{\odot}$ model with an initial period of 10 days and no disk-locking. The value of $\omega_{\text {crit }}$ has been found to depend on mass (Krishnamurthi et al. 1997, Barnes \& Sofia 1996). We have used the prescription for the variation of $\omega_{\text {crit }}$ with mass from Krishnamurthi et al. (1997). In this prescription, $\omega_{\text {crit }}$ is inversely proportional to the convective overturn timescale in the star at 200 Myr (Kim \& Demarque 1996):

$$
\omega_{\text {crit }}=\omega_{\text {crit } \odot} \frac{\tau_{\odot}}{\tau}
$$

The convective overturn times were linearly extrapolated for masses lower than $0.5 \mathrm{M}_{\odot}$. We have also considered models in which no angular momentum loss is allowed, to determine the maximum structural effects of rotation.

We have calculated the rotational evolution of two classes of stellar models: models in which solid body rotation is enforced at all time, and models in which internal angular momentum transport is affected by hydrodynamic mechanisms. The rotation rate of the solid body models is determined from the moment of inertia of the model at a given time, and the total angular momentum as determined by the loss rate given in equation 8 . For the second set of models, rigid rotation is enforced throughout the convection zone only, and the rotation in the interior is governed by the transport of angular momentum by hydrodynamic mechanisms. The chemical mixing associated with this angular momentum transport is computed using a set of diffusion equations (Pinsonneault et al. 1989); the amount of coupling between transport and mixing is calibrated by requiring that the amount of lithium depletion calculated by our model matches the observed value for the Sun.

We start our models on the birthline of Palla \& Stahler (1991), which is the deuteriumburning main sequence and corresponds to the upper envelope of $\mathrm{T}$ Tauri observations in 
the HR diagram. It has been shown (Barnes \& Sofia 1996) that this physically realistic assumption for the initial conditions of stellar rotation models is crucial for accurate modeling of ultra-fast rotators in young clusters. The models with no angular momentum loss began with an initial rotation period of 8 days, corresponding to the mean classical T Tauri star rotation period (Choi \& Herbst 1996). The models which included angular momentum loss began with initial rotation periods of either 4 or 10 days. We present models for solar metallicity stars between 0.1 and $1.1 \mathrm{M}_{\odot}$ in increments of $0.1 \mathrm{M}_{\odot}$. These models have been evolved from the birthline to an age of 10 Gyr.

\section{Results}

\subsection{The Structural Effects of Rotation}

Evolutionary tracks for both rotating and non-rotating models are presented in figure 1. The rotating models have initial periods of 10 days and experience no angular momentum loss. As expected (Sackmann 1970, Pinsonneault et al. 1989), the effect of rotation is to shift stars to lower effective temperatures and lower luminosities, mimicking a star of lower mass. This effect is most pronounced for the highest mass stars presented in this paper, and is reduced to a low level for stars less than $0.4 \mathrm{M}_{\odot}$. Since low mass stars are fully convective, their temperature gradient will be the adiabatic gradient, which does not depend on the rotation rate (equation 7). However, the structural effects of rotation are still apparently in fully convective stars, and diminish with decreasing mass. This suggests that an additional mechanism is also at work. As stars get less massive, their central pressure is being provided less by thermal pressure and more by degeneracy pressure. The amount of degeneracy is determined by the density in the interior, which is not affected by rotation (see equation 1). Rotation provides an additional method of support for the star, but in stars with a significant amount of degeneracy, the rotational support is a smaller fraction of the total pressure. Therefore, the structure of the low mass stars is less affected by rotation than their higher mass counterparts.

Figure 2 compares the evolutionary tracks for rotating stars under different assumptions about internal angular momentum transport. The solid tracks are stars which have differentially rotating radiative cores and rigidly rotating convection zones, while the dashed lines show the tracks for stars which are constrained to rotate as solid bodies. The two tracks for each mass have the same surface rotation rate at the zero-age main sequence. The low mass stars show no difference between the two assumptions, since these stars are fully convective for the entire 10 Gyr plotted here. Therefore, they always rotate as solid bodies. The higher mass stars begin their lives high on the pre-main sequence as fully 
convective, solid body rotators. As they contract and develop radiative cores, however, the difference in the two assumptions about angular momentum transport becomes apparent. Differential rotators have a higher total angular momentum than solid body rotators of the same surface rotation rate. As stars contract along the pre-main sequence, they become more centrally concentrated, which means that the core spins up more than the envelope does. The solid body rotators are forced to spread their angular momentum evenly throughout the star, so they have less total angular momentum for a given surface rotation rate. Therefore, the impact of rotation on the structure of the star is larger for differential rotators than for solid body rotators of the same surface rotation rate. However, at constant initial angular momentum, the solid body rotators are cooler at the zero age main sequence, and have longer pre-main sequence lifetimes, than differentially rotating stars of the same mass. When comparing the effects of rotation between different models, it is important to note whether the comparison is between stars with the same current surface rotation rate, or with the same initial angular momentum.

We included the kinetic energy of rotation $\left(T=\frac{1}{2} I \omega^{2}\right)$ in our determination of the total luminosity in each shell of the star. As the star changes its moment of inertia $I$ and its rotation rate $\omega$, the resulting change in its rotational kinetic energy can be included in the energy budget of the star. Most implementations of stellar rotation into stellar structure and evolution neglect this energy since it is expected that the amount of kinetic energy available is not enough to significantly affect the evolution of the star. Since very low mass stars have much lower luminosities than solar-mass stars, but their moments of inertia are not as significantly lower, it is plausible that the kinetic energy of rotation would contribute a significant fraction of the total luminosity of the star. As shown in figure 4 , however, the change in the kinetic energy of rotation contributes no more than $6 \%$ of the total luminosity of the star in the $1.0 \mathrm{M}_{\odot}$ model, and that contribution lasts less than $50 \mathrm{Myr}$. As expected, the lowest mass star has the most significant contribution, lasting for about 1 Gyr, but at $4 \%$ or less. The positions of stars in the HR diagram are minimally affected by the inclusion of this source of energy. The kinetic energy of rotation reduces the luminosity at any given time by less than 0.02 dex in $\log \left(L / L_{\odot}\right)$, and usually less than 0.005 dex. The timescales for evolution are also equally unaffected. The models with no angular momentum loss will have the maximum possible effect of rotational kinetic energy. Since these models show no significant effect, we conclude that the change in the kinetic energy of rotation is at most a perturbation on the structure.

The main structural effect of rotation is a reduction in the effective temperature of stars. Using our tracks, we have quantified the relationship between rotational velocity and the difference in effective temperature at the zero age main sequence. In figure 5 we present this relationship for stars of different masses, and for both the differentially rotating 
(solid lines) and solid body models (dashed lines). For low mass stars, the difference in temperature caused by rotation is of order a few tens of $K$ (and reduces to less than 10 $\mathrm{K}$ for stars of $0.2 \mathrm{M}_{\odot}$ ). This difference is therefore negligible. However, the reduction in effective temperature is larger for the more massive stars, and can reach significant levels of a few hundred $\mathrm{K}$ for stars more massive than about $0.6 \mathrm{M}_{\odot}$. Therefore, when determining masses from observed temperatures or colours, it is important know how fast these stars are rotating. The relationship between rotation rate and difference in effective temperature, for a given stellar mass, is well-fit by a polynomial. The coefficients for this polynomial at different masses and under different assumptions of internal angular momentum transport are given in table 2. It should be noted that while solid body rotators of the same initial period rotate faster at the zero age main sequence than differentially rotating stars, the structural effects of rotation are slightly more pronounced in the differential rotators at constant surface rotation speed. Therefore, for a constant rotational velocity, stars which rotate differentially have a higher angular momentum than solid body rotators.

For stars of the same mass, rotation reduces the luminosity of stars as well as their temperatures. The difference in luminosity is not as important as the difference in temperature cause by rapid rotation, as shown in figure 5. Even for the most extreme case, the difference in luminosity for a $1.0 \mathrm{M}_{\odot}$ star rotating at $250 \mathrm{~km} / \mathrm{s}$ is less than $0.12 \mathrm{dex}$ in $\log \left(L_{\odot}\right)$. While differences of this size will result in a thicker main sequence of a cluster, it should not affect any scientific results significantly. Most stars in clusters do not rotate very fast, so the upper main sequence will be well-defined for any isochrone fitting or distance determination. Luminosity is used as an indicator of mass for low mass stars, but since the difference in luminosity between rapid rotators and non-rotators is very small for low mass stars, this calibration should not be affected by rotation.

The total effect of rotation is such that the locus of the zero age main sequence becomes brighter as stars rotate more quickly. The combination of a significant decrease in temperature with a small decrease in luminosity for stars of the same mass moves the locus above the non-rotating main sequence. At a surface rotation rate of $100 \mathrm{~km} / \mathrm{s}$, the rotating main sequence is brighter by about 0.01 magnitudes. At $200 \mathrm{~km} / \mathrm{s}$, the sequence is brighter by 0.03 magnitudes. Therefore, we expect to see rapid rotators in clusters lying above the cluster main sequences by a few hundredths of a magnitude.

Since they are fainter, rapid rotators have slightly longer lifetimes compared to non-rotating stars of the same mass. The amount of increase depends on the mass of the star and the rotation rate, but in the most extreme case $\left(1.0 \mathrm{M}_{\odot}\right.$ rotating at $\left.250 \mathrm{~km} / \mathrm{s}\right)$, the difference in pre-main sequence lifetime is $7 \%$. For rotation rates less than $100 \mathrm{~km} / \mathrm{s}$, the increase in lifetime is less than $1 \%$ for all masses. 


\subsection{Rotational Evolution}

We have compared our models with rotational data from young open clusters. Each cluster provides a sample of stars with different masses, allowing us to study the effects of mass on angular momentum loss and disk lifetimes. By comparing the progression of rotation rates from very young clusters to older ones, we can study the rotational history of stars of a range of masses. Both probes are very useful in the study of stellar rotation. There are four well-studied data sets used when investigating rotation in young open clusters: IC 2602 and IC 2391, at 30 Myr; $\alpha$ Persei, at 60 Myr, the Pleiades at $110 \mathrm{Myr}$; and the Hyades, at 600 Myr. In figures 6-16, we compare our models to observational data at the appropriate age for the cluster, under a number of different assumptions about the initial conditions, the internal transport of angular momentum, the saturation threshold, and the disk locking lifetime. The data were taken from Stauffer et al. 1997b (IC 2602 and IC 2391), Prosser et al. 1995 and references therein ( $\alpha$ Per), Soderblom et al. 1993; Prosser et al. 1995 and Stauffer et al. 1999 (Pleiades and Hyades), and Radick et al. 1987 (Hyades), supplemented with data from the Open Cluster Database (Prosser \& Stauffer 1999).

We wish to reproduce a number of important features of these data sets. First, our models must predict the correct rotation rates for the fast rotators in each of these clusters. The presence of fast rotators is caused by the saturation of the angular momentum loss law (equation 8), and constrains the value of $\omega_{\text {crit }}$ as a function of mass. Also, our models should reproduce the spin-down of the fast rotators with time, as seen by comparing stars of the same mass in different clusters.

The comparison between our models and observed fast rotators confirms the conclusion of Krishnamurthi et al. (1997) that a mass-dependent $\omega_{\text {crit }}$ is necessary, in the sense that $\omega_{\text {crit }}$ increases for increasing mass. Figures 6 and 7 present rotational models with

different normalizations of the Rossby scaling and initial periods of 10 days. Figure 6 presents models which rotate as solid bodies throughout their lifetime, while figure 7 shows differentially rotating models in which internal angular momentum transport is determined by hydrodynamical instabilities. The thick solid lines in each frame represent the upper envelope of rotation rates observed in each cluster, which have not been corrected for inclination angle. Observations of X-ray activity in rotating stars as a function of $v \sin i$ show that reasonable values for the angular momentum loss saturation threshold velocity range from 5 to $20 \omega_{\odot}$ (Patten \& Simon 1996). We have plotted three different normalizations, with values of $\omega_{\text {crit } \odot}=5,10$ and $20 \omega_{\odot}$ (from top to bottom each frame). For the solid body models (figure 6), it is clearest at the older ages that stars with a high saturation threshold lose too much angular momentum, and the low saturation threshold models lose too little. Therefore, the best choice for normalization is about $10 \omega_{\odot}$. For the 
differentially rotating models, the best choice for normalization is about $5 \omega_{\odot}$, since the

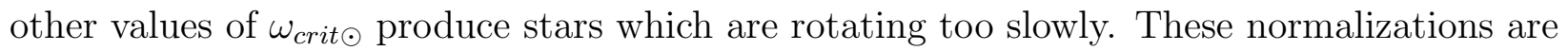
the same as the ones adopted by Krishnamurthi et al. (1997) for solar analogues.

The Rossby scaling with the normalizations suggested by Krishnamurthi et al. (1997) are shown in figures 8 (solid body models) and 9 (differentially rotating models). The upper line in each frame corresponds to models with initial rotation periods of 4 days, while the lower line corresponds to models with initial periods of 10 days. The fastest rotators in all the clusters lie below the 4 day line, with the exception of the lowest mass stars in the Hyades, which rotate faster than the predictions of the differentially rotating models. We conclude that a different normalization for the Rossby scaling is necessary for low mass stars.

The second main feature of these data sets that we wish to reproduce is the large spread in rotation rate at constant mass. This range in rotational velocity is caused by a range in protostellar disk lifetime. In figures 10 and 11, we present models with initial rotation periods of 10 days, and the above-mentioned normalizations for $\omega_{\text {crit }}$. The upper line in each frame shows models which have detached from their disk at the birthline. Moving down each frame, the lines represent models with disk lifetimes of $0.3,1,3$ and $10 \mathrm{Myr}$ from the birthline. The differentially rotating models (figure 11) reproduce the rotation rates of the slowest rotators in these clusters with disk lifetimes of 10 Myr or less, while the solid body models (figure 10) require longer disk lifetimes, perhaps even as long as the current age of the cluster.

We have verified that neither the Krishnamurthi et al. (1997) Rossby scaling nor any other unique Rossby scaling can reproduce the mass dependence of the angular momentum evolution below $4000 \mathrm{~K}$, corresponding to masses of $0.6 \mathrm{M}_{\odot}$ and below. The persistence of rapid rotation in the Hyades cluster requires inefficient angular momentum loss in the lowest mass stars, while a uniform lowering of the saturation threshold would predict more rapid rotation for higher mass stars than is observed in the Hyades. We therefore constructed models for the 0.1 to $0.5 \mathrm{M}_{\odot}$ range where the value of $\omega_{\text {crit }}$ was tuned to reproduce the Hyades upper envelope; we adopted the Krishnamurthi et al. (1997) Rossby scaling for the higher mass models.

Figures 12 and 13 show the same models as in figures 10 and 11, but with this different normalizations of the mass dependence for $\omega_{\text {crit }}$. In figure 12 , we present solid body rotational models with $\omega_{\text {crit } \odot}=7,6.2,5.1,3.6,1.7 \omega_{\odot}$ at $0.5,0.4,0.3,0.2$ and $0.1 \mathrm{M}_{\odot}$ respectively. Figure 13 shows differentially rotating models with $\omega_{\text {crit } \odot}=3,3.3$, 3.5, 1.9, $0.9 \omega_{\odot}$ for the same masses. Both these sets of models reproduce the fastest rotators in the Hyades at low masses. With the exception of a different zero-point for the 
saturation threshold, the solid body models and the differentially rotating models predict very similar angular momentum evolution histories for these low mass stars. The different normalization and similar evolution can be understood as follows. The DR models require a higher constant in the angular momentum loss law to extract angular momentum from a rapidly rotating core and a slowly rotating envelope. For models with $\omega>\omega_{\text {crit }}$, the angular momentum loss rates can be made identical by a suitable zero-point shift in $\omega_{\text {crit }}$. Although the DR models would experience more severe angular momentum loss for $\omega<\omega_{\text {crit }}$, the low values of $\omega_{\text {crit }}$ required for reproducing the stellar rotation data are not reached until ages older than the clusters that we are studying.

In figure 14 we use the models presented in the previous two figures to produce distributions of disk lifetimes for three of the clusters studied in this paper. A statistical correction of $4 / \pi$ was applied to the observed rotation velocities. This overestimates the rotation rates for the rapid rotators, where $\sin i$ is likely to be close to one, but does provide a more accurate estimate on average for the bulk of the slow rotator population. We find an essentially constant distribution of disk lifetimes with age for the differentially rotating models across the young clusters. Disk lifetimes longer than $10 \mathrm{Myr}$ are inconsistent with observations of infrared excesses around T Tauri stars (Strom et al. 1989), which predict maximum disk lifetimes between 3 and 10 Myr. Therefore, the large fraction of stars which are required to have long disk lifetimes is an argument against the solid body models.

The predicted rotation rates at the age of the Hyades are systematically slightly higher than the data for the differentially rotating models at the higher end of the mass range, while the solid body models are in good agreement with the observed range. This leads to spuriously short disk lifetimes for the higher mass stars when combined with the insensitivity of the rotation to the initial conditions at this late age. We interpret this as an indication that the angular momentum coupling time scale is intermediate between the Pleiades and Hyades ages, which is consistent with the flat solar rotation curve.

The recent Orion data set of Stassun et al. (1999) also has interesting consequences for angular momentum evolution. They were sensitive to rotation periods shorter than eight days; this complicates the question of directly testing the accretion disk-locking model, since this is near the peak of the period distribution found by earlier studies of young stars. However, they found 85 (out of 264) stars with rotation periods less than 3 days. By comparison, models with an initial rotation period of eight days would have a rotation period of 2.64 days at an age of 1 Myr. Stassun et al. (1999) observed a cutoff in the distribution below 0.5 days, which would correspond to a rotation period of 2 days at the birthline (a factor of two greater than the maximum angular momentum we assumed for setting the upper envelope of the distribution). This indicates that both the initial 
period and the distribution of accretion disk lifetimes needs to be taken into account when modeling the rapid rotator distribution. Because we use the upper envelope of rotation to set the value of $\omega_{\text {crit }}$, assigning a shorter initial period to the upper envelope of rotation would imply systematically larger values of $\omega_{\text {crit }}$. This would lead to shorter predicted disk lifetimes for the slower rotators in young open clusters, although not enough to alter the qualitative conclusions about differentially rotating versus solid body models.

An additional feature of these models is the possible lack of long disk lifetimes observed for very low mass stars. This is most obvious in a plot of disk lifetime as a function of $\mathrm{T}_{\text {eff }}$, shown in figures 15 and 16. Both solid body and differentially rotating models show the same trend. In the Pleiades, we would expect to see stars with $v \sin i$ at or near the lower detection limit of $7 \mathrm{~km} / \mathrm{s}$ below $3500 \mathrm{~K}$. The low mass portion of the data set was chosen based on colour of the stars and not on any rotational information, and therefore should be unbiased (Stauffer et al. 1999). However, we see a lack of slow rotators at the low mass end.

There are two possible explanations. One scenario is that the lowest mass stars have systematically shorter accretion disk lifetimes than higher mass stars. A second possibility is suggested by a comparison of figures 10 and 11 with figures 12 and 13; the mass dependence of the saturation threshold for angular momentum loss has a strong influence on the predicted rotation rates for a given initial condition. With the small Hyades cool star sample, the upper envelope appears to be flat; we therefore infer relatively short disk coupling lifetimes for the lowest mass stars. However, if the upper envelope were to rise with decreasing mass in the Hyades this would indicate that it is the angular momentum loss law, not a mass-dependent set of initial conditions, that was responsible. We cannot rule this explanation out due to the relatively small sample of Hyades stars cooler than 3500 K. However, the two solutions make different predictions for the observed rotation rates in older clusters. If the trend towards higher mean rotation rates at lower mass in the Hyades persists below $3500 \mathrm{~K}$, this indicates that the mass dependence of the angular momentum loss law is the primary cause; if the data can be fit using the existing angular momentum

loss law with consistently shorter disk lifetimes for lower mass stars it is an indication of a genuine change in the distribution of initial conditions as a function of mass.

\section{Discussion}

In this paper, we present models of very low mass stars $\left(<0.5 \mathrm{M}_{\odot}\right)$ which include the effects of rotation. These models have been made possible by the work of a number of groups on the physics of low temperature stellar atmospheres, opacities and equations of state. By including the physics of many molecules in stellar atmosphere calculations, 
Allard \& Hauschildt (1995) have created models which are valid for low mass stars with effective temperatures less than $4000 \mathrm{~K}$. The equation of state of Saumon, Chabrier \& Van Horn (1995) includes partial dissociation and ionization of hydrogen and helium caused by both pressure and temperature effects, and is applicable to both low mass stars and giant

planets. Finally, Alexander \& Ferguson (1994) added atomic line absorption, molecular line absorption and some grain absorption and scattering to the usual sources of continuous opacity to produce opacity tables which reach to temperatures as low as $700 \mathrm{~K}$. Since most previous atmosphere, opacity and equation of state did not include the effects of molecules and grains, these three improvements represent a great leap forward in our ability to model very low mass stars.

\subsection{The Structural Effects of Rotation}

We have investigated the effect of rotation on the structure of low mass stars. We discussed a number of implications, based on models which demonstrate the maximum extent of the differences between rotating and non-rotating models. The most important effect is the reduction of effective temperature for stars of a given mass. Rapid rotators are cooler than slow rotators, and so, for stars more massive than $0.5 \mathrm{M}_{\odot}$, any relationship between temperature and mass should take into account the rotation rate of the star. We have shown that the structural effects of rotation in very low mass stars (less than $\sim 0.5$ $\left.\mathrm{M}_{\odot}\right)$ are minimal and can be neglected when interpreting temperatures and luminosities of these stars from observations. Table 2 gives the polynomial correction between the effective temperature of a rotating star and that of a non-rotating star, as a function of rotation rate and stellar mass.

We have shown that the kinetic energy of rotation is not a significant contribution to the total luminosity of stars between 0.1 and $1.0 \mathrm{M}_{\odot}$, and does not change the timescale for evolution on the pre-main sequence. This rotational contribution to the total energy of the star does not affect the position of the star in the HR diagram, and we have neglected this effect in the evolutionary calculations presented in this paper.

Stellar activity can also influence the colour-temperature relationship; because of the well-known correlation between increased rotation, increased stellar spot coverage, and increased chromospheric activity this will tend to change the observed position of rapidly rotating stars in the HR diagram relative to slow rotators. Different colour indices are affected to different degrees; for example, Fekel, Moffett, \& Henry (1986) found a systematic departure between the B-V and V-I colours of active stars. Stars with more modest activity levels have a more normal colour-colour relationship (e.g. Rucinski 1987). Active stars tend 
to be bluer in B-V than in V-I relative to less active stars. Fekel, Moffett, \& Henry (1986) treated this as an infrared excess, but in open clusters such as the Pleiades and $\alpha$ Persei rapid rotators are on or above the main sequence in $\mathrm{V}-\mathrm{I}$, but can be below the zero-age main sequence in B-V (Pinsonneault et al. 1998). Given the theoretical trends presented here, this suggests that V-I is a good tracer of temperature, and that B-V is the colour which is most affected by activity. The difference between effective temperatures based on B-V and those based on V-I can reach $200 \mathrm{~K}$ in Pleiades stars (Krishnamurthi, Pinsonneault, King, \& Sills 1999).

\subsection{Angular Momentum Evolution}

The study of low mass stars can provide valuable constraints on the three coupled ingredients of angular momentum evolution models: internal angular momentum transport, angular momentum loss, and the distribution of initial conditions.

We have compared the properties of solid body models with those of models with internal angular momentum transport from hydrodynamic mechanisms. We confirm previous results that the angular momentum evolution of systems at and younger than the Pleiades age of $110 \mathrm{Myr}$ are best reproduced by models which permit differential rotation with depth. We also find that the solid-body models do a better job of reproducing the data at the Hyades age (600 Myr) and older; in addition, helioseismic data are inconsistent with the strong differential rotation with depth predicted by models with hydrodynamic angular momentum transport. The simplest solution that is consistent with the data is an additional angular momentum transport mechanism with a time scale intermediate between $110 \mathrm{Myr}$ and $600 \mathrm{Myr}$.

Angular momentum loss has a strong impact on the rotational history of low mass stars; the greatest challenge in understanding the angular momentum evolution of low mass stars has been distinguishing between the effects of angular momentum loss, internal angular momentum transport, and the initial conditions. The combination of deep convective envelopes and mild angular momentum loss in stars below $0.6 \mathrm{M}_{\odot}$ makes their behaviour

insensitive to the treatment of internal angular momentum transport. We believe that these stars provide a simpler laboratory for the study of the two major other ingredients, namely the loss law and the initial conditions.

Previous work established that an angular momentum loss law which saturates at a mass-dependent critical value of the stellar angular velocity is required to reproduce the fastest rotators in young clusters. We find that the prescription of Krishnamurthi et al. 
(1997), in which $\omega_{\text {crit }}$ is inversely proportional to the convective turnover time of the star at 200 Myr (the Rossby scaling), yields a consistent solution from 0.6 to $1.1 \mathrm{M}_{\odot}$. A Rossby scaling underestimates the mass dependence when extended to the lowest mass stars, in the sense that the efficiency of angular momentum loss drops more rapidly than predicted by a Rossby scaling. Models for stars below $3500 \mathrm{~K}\left(\sim 0.4 \mathrm{M}_{\odot}\right)$ do not yield consistent initial conditions compared to more massive stars even when the normalization appropriate for the observed upper envelope of the Hyades data is used. It is possible that this could reflect a change in the distribution of initial conditions, but more data are required to rule out the angular momentum loss rate as a cause. It is also possible that our estimate of the global convective overturn time is inaccurate for the lowest mass stars. However, it is clear that angular momentum loss must be occurring, since models which experience no angular momentum loss are rotating at velocities which are at least a factor of 2 too large to agree with any of the observations. In general, we find no evidence for a significant change in the form of angular momentum evolution and loss when stars become fully convective; all of the trends observed are smooth as a function of mass without a sharp break at the fully convective boundary.

This last point has implications for cataclysmic variable (CV) research. The orbital periods of these mass-accreting white dwarfs are observed to have a gap between 2 and 3 hours, in which very few systems are seen. The accepted interpretation for this gap has been a sharp reduction in angular momentum loss rate as the stars become fully convective (see Patterson 1984 for a complete introduction to CVs, and McDermott \& Taam 1989 for one particular $\mathrm{CV}$ model). For masses higher than $\sim 0.3 \mathrm{M}_{\odot}$, angular momentum loss through a magnetic wind (magnetic braking) is assumed to occur in the mass-losing secondary of the CV system. The angular momentum is lost from the system entirely, and the secondary continues to fill its Roche lobe, transferring mass onto the white dwarf. This mass transfer keeps the secondary out of thermal equilibrium, so the star has a slightly larger radius for its mass than an isolated star. When the star becomes fully convective, however, it is proposed that the secondary's magnetic field is no longer anchored to the radiative core of the star, and abruptly ceases to exist. Angular momentum (and hence mass) is no longer transferred from the secondary, and the secondary is allowed to contract to its normal main sequence radius. Only when the system shrinks further, due to gravitational radiation, does the mass transfer restart, and the system again becomes a CV. We have not seen any sharp break in the angular momentum loss rates as we move to lower masses, suggesting that any theory for the period gap of cataclysmic variables which relies on the cessation of angular momentum loss requires a mechanism other than the standard magnetic braking.

There are several promising directions for future theoretical studies. First, there is an increasing database of rotational periods and velocities in systems with a range 
of ages and metal abundances. A combined analysis of the information in protostars, young clusters, and intermediate-aged systems will provide interesting constraints on theoretical models. The next generation of theoretical models should incorporate multiple physical mechanisms for internal angular momentum transport while also relying on the complementary information on surface mixing. More sophisticated models of stellar winds and angular momentum loss will also be needed to investigate the physical implications of the empirical trends deduced in theoretical studies of the type we have performed.

We would like to conclude with a plea to observers of young open clusters. The observational database for rotation rates of very low mass stars is quite sparsely populated. We have observations of stellar rotation rates down to about $0.2 \mathrm{M}_{\odot}$ in the Pleiades and Hyades, but the data in $\alpha$ Persei, IC 2602 and IC 2391 have only spotty coverage below $0.6 \mathrm{M}_{\odot}$. These young stars provide constraints on the early spindown of low mass stars. The Hyades is the oldest cluster in this sample, and many of the different scenarios are best distinguished from each other at later ages. It would be very beneficial to this field to determine rotation rates for the lower mass stars in young open clusters.

This work was supported by NASA grant NAG5-7150. A. S. wishes to recognize support from the Natural Sciences and Engineering Research Council of Canada. We would like to acknowledge use of the Open Cluster Database, as provided by C.F. Prosser (deceased) and J.R. Stauffer, and which currently may be accessed at http://cfawww.harvard.edu/ stauffer/, or by anonymous ftp to cfa0.harvard.edu (131.142.10.30), cd /pub/stauffer/clusters/.

\section{REFERENCES}

Alexander, D. R., \& Ferguson, J. W. 1994, ApJ, 437, 879

Allain, S. 1998, A\&A, 333, 629

Allard, F., \& Hauschildt, P. H. 1995, ApJ, 445, 433

Baraffe, I., Chabrier, G., Allard, F., \& Hauschildt, P. H. 1998, A\&A, 337, 403

Barnes, G., Charbonneau, P., \& MacGregor, K.B. 1999, ApJ, 511, 466

Barnes, S., \& Sofia. S. 1996, ApJ, 462, 746

Barnes, S., Sofia. S., Prosser, C. F., \& Stauffer, J. R. 1999, ApJ, 516, 263

Böhm-Vitense, E. 1958, Zs. f. Ap., 46, 108

Bouvier, J., Forestini, M., \& Allain, S. 1997, A\&A, 326, 1023 
Chaboyer, B., Demarque, P., \& Pinsonneault, M. H. 1995, ApJ, 441, 865

Chaboyer, B., \& Zahn, J.-P. 1992, A\&A, 253, 173

Charbonneau, P. \& MacGregor, K.B. 1993, ApJ, 417, 762

Choi, P. I., \& Herbst, W. 1996, AJ, 111, 283

Collier-Cameron, A.C., Campbell, C.G., \& Quaintrell, H. 1995, A\&A, 298, 133

Collier-Cameron, A.C. \& Jianke, L. 1994, MNRAS, 269, 1099

Cox, J. P. \& Guili, R. T. 1968, Principles of Stellar Structure, in two volumes (New York: Gordon and Breach)

Edwards, S., Strom, S. E., Hartigan, P., Strom, K. M., Hillenbrand, L. A., Herbst, W., Attridge, J., Merrill, K. M., Probst, R., \& Gatley, I. 1993, AJ, 106, 372

Endal, A. S., \& Sofia, S. 1976, ApJ, 210, 184

Fekel, F. C., Moffett, T. J., \& Henry, G. W. 1986, ApJS, 60, 551

Grevesse, N., \& Noels, A. 1993, in Origin and Evolution of the Elements, ed. N. Prantzos, E. Vangioni-Flam, \& M. Cassé (Cambridge:Cambridge Univ. Press), 15

Gruzinov, A., \& Bahcall, J. 1998, ApJ, 504, 996

Guenther, D. B., Demarque, P., Kim, Y.-C., \& Pinsonneault, M. H. 1992, ApJ, 387, 372

Hartmann, L. W., \& Noyes, R. N. 1987, ARA\&A, 25, 271

Iglesias, C. A., \& Rogers F. J., 1996 ApJ, 464, 943

Kawaler, S. D. 1988, ApJ, 333, 236

Keppens, R., MacGregor, K.B., \& Charbonneau, P. 1995, A\&A, 294, 469

Kippenhahn, R., \& Thomas, H.-C. 1970 in Stellar Rotation, ed. A. Slettebak (Dordrecht: Reidel), p. 20

Kim, Y.-C., \& Demarque, P. 1996, ApJ, 457, 340

Königl, A. 1991, ApJ, 370, L39

Kraft, R. 1965, ApJ, 142, 681

Krishnamurthi, A., Pinsonneault, M. H., Barnes, S., Sofia, S. 1997, ApJ, 480, 303

Krishnamurthi, A., Terndrup, D. M., Pinsonneault, M. H., Sellgren, K., Stauffer, J. R., Schild, R., Backman, D. E., Beisser, K. B., Dahari, D. B., Dasgupta, A., Hagelgans, J. T., Seeds, M. A., Anand, R., Laaksonen, B. D., Marschall, L. A., Ramseyer, T. 1998, ApJ, 493, 914

Krishnamurthi, A., Pinsonneault, M. H., King, J. R., \& Sills, A., 1999, in preparation 
McDermott, P. N. \& Taam, R. E. 1989, ApJ, 342, 1019

Meynet, G., \& Maeder, A. 1997, A\&A, 321, 465

Noyes, R.W., Hartmann, L.W., Baliunas, S.L., Duncan, D.K., \& Vaughn, A.H. 1984, ApJ, 279, 763

Palla, F., \& Stahler, S. W. 1991, ApJ, 375, 288

Patterson, J. 1984, ApJS, 54, 443

Patten, B., \& Simon, T. 1996, ApJS, 106, 489

Pinsonneault, M. H. 1997, ARA\&A, 35, 55

Pinsonneault, M. H., Kawaler, S. D., Sofia, S., \& Demarque, P. D. 1989, ApJ, 338, 424

Pinsonneault, M.H., Kawaler, S.D., \& Demarque, P. 1990, ApJS, 74, 501

Pinsonneault, M. H., Stauffer, J., Soderblom, D. R., King, J. R., \& Hanson, R. B. 1998 ApJ, 504, 170

Prosser, C. F., Shetrone, M. D., Dasgupta, A., Backman, D. E., Laaksonen, B. D., Baker, S. W., Marschall, L. A., Whitney, B. A., Kuijken, K., \& Stauffer, J. R. 1995, PASP, 107,211

Prosser, C. F., \& Stauffer, J. 1999 The Open Cluster Database, http://cfawww.harvard.edu/ stauffer/opencl/index.htm]

Radick, R. R., Thompson, D. R., Lockwood, G. W., Duncan, D. K., \& Bagett, W. E. 1987, ApJ, 321, 459

Rogers, F. J., Swenson, F. J., \& Iglesias, C. A. 1996, ApJ, 456, 902

Rucinski, S. M. 1987, PASP, 99, 288

Sackman, I.J. 1970, A\&A, 8, 76

Santiago, B., Elson, R., \& Gilmore, G. 1996, MNRAS, 281, 1363

Saumon, D., Chabrier, G., \& Van Horn, H. M. 1995, ApJS, 99, 713

Soderblom, D. R., Stauffer, J. R., MacGregor, K. B., \& Jones, B. F. 1993, ApJ, 409, 624

Stassun, K. G., Mathieu, R. D., Mazeh, T., \& Vrba, F. J. 1999, AJ, 117, 2941

Stauffer, J. R., \& Hartmann, L. W. 1987, ApJ, 318, 337

Stauffer, J. R., Balachandran, S. C., Krishnamurthi, A., Pinsonneault, M., Terndrup, D. M., Stern, R. A. 1997a, ApJ, 475, 604

Stauffer, J. R., Hartmann, L. W., Prosser, C. F., Randich, S., Balachandran, S., Patten, B. M., Simon, T., \& Giampapa, M. 1997b, ApJ, 479, 776 
Stauffer, J. R., Schultz, G., Kirkpatrick, J. D. 1998, ApJ, 499, L199

Stauffer, J. R., Terndrup, D. M., Pinsonneault, Sills, A., Yuan, Y., Jones, B. F., Fischer, D., Krishnamurthi, A. 1999, in preparation

Strom, K. M., Strom, S. E., Edwards, S., Cabrit, S., \& Skrutskie, M. F. 1989, AJ, 97, 1451

Talon, S. \& Zahn, J.-P. 1998, A\&A, 329, 315

Tinney, C. G., Mould, J. R., \& Reid, I. N. 1993, AJ, 105, 1045

von Hippel, T., Gilmore, G., Tanvir, N., Robinson, D., \& Jones, D. H. P. 1996, AJ, 112, 192

Weber, E. J., \& Davis, L., Jr. 1967, ApJ, 148, 217 


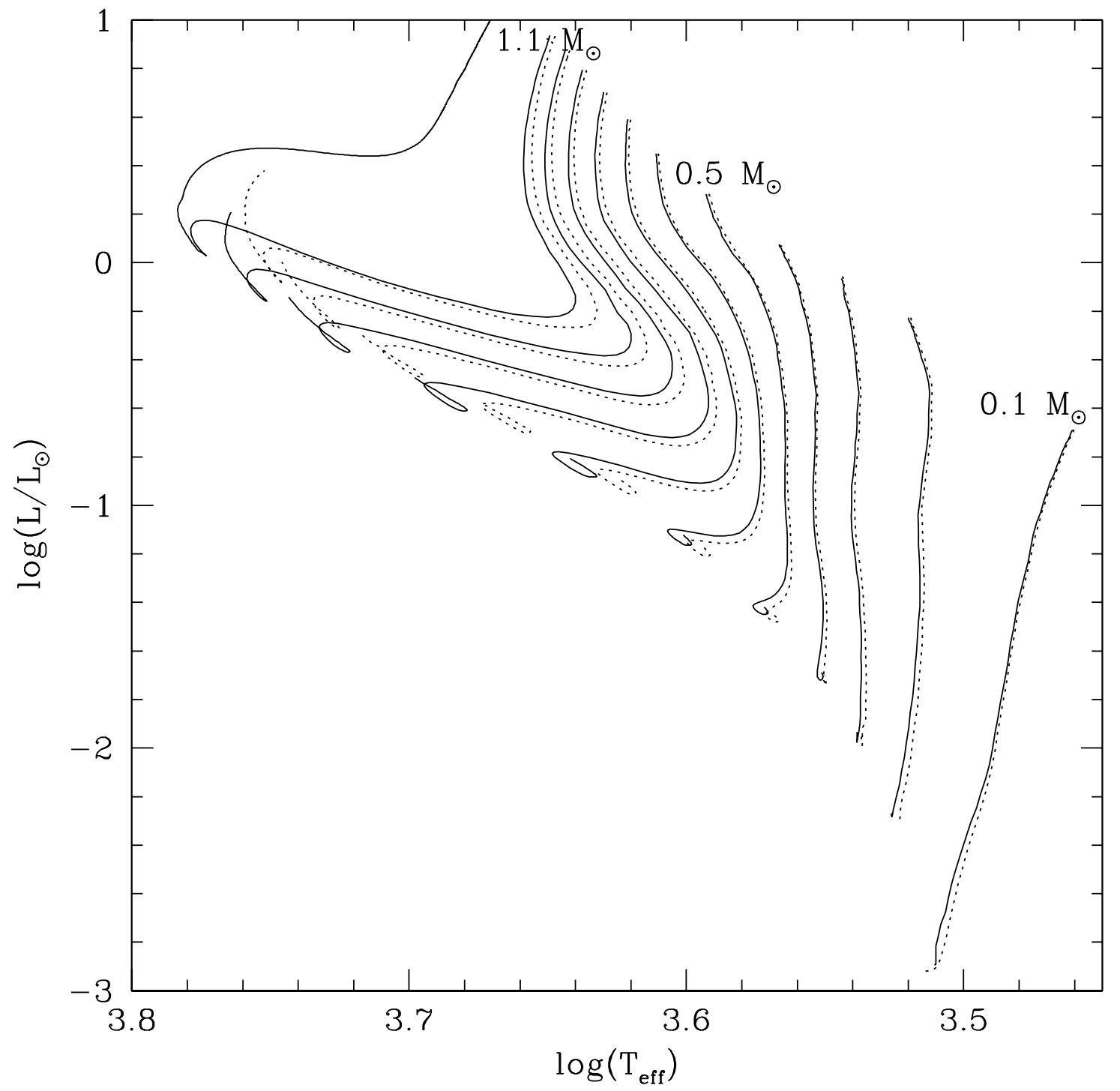

Fig. 1. - Evolutionary tracks for stars with masses between $0.1 \mathrm{M}_{\odot}$ and $1.1 \mathrm{M}_{\odot}$ in steps of $0.1 \mathrm{M}_{\odot}$. The solid lines are stars without rotation, and the dotted lines are stars which have initial rotation periods of 8 days, and no angular momentum loss. 


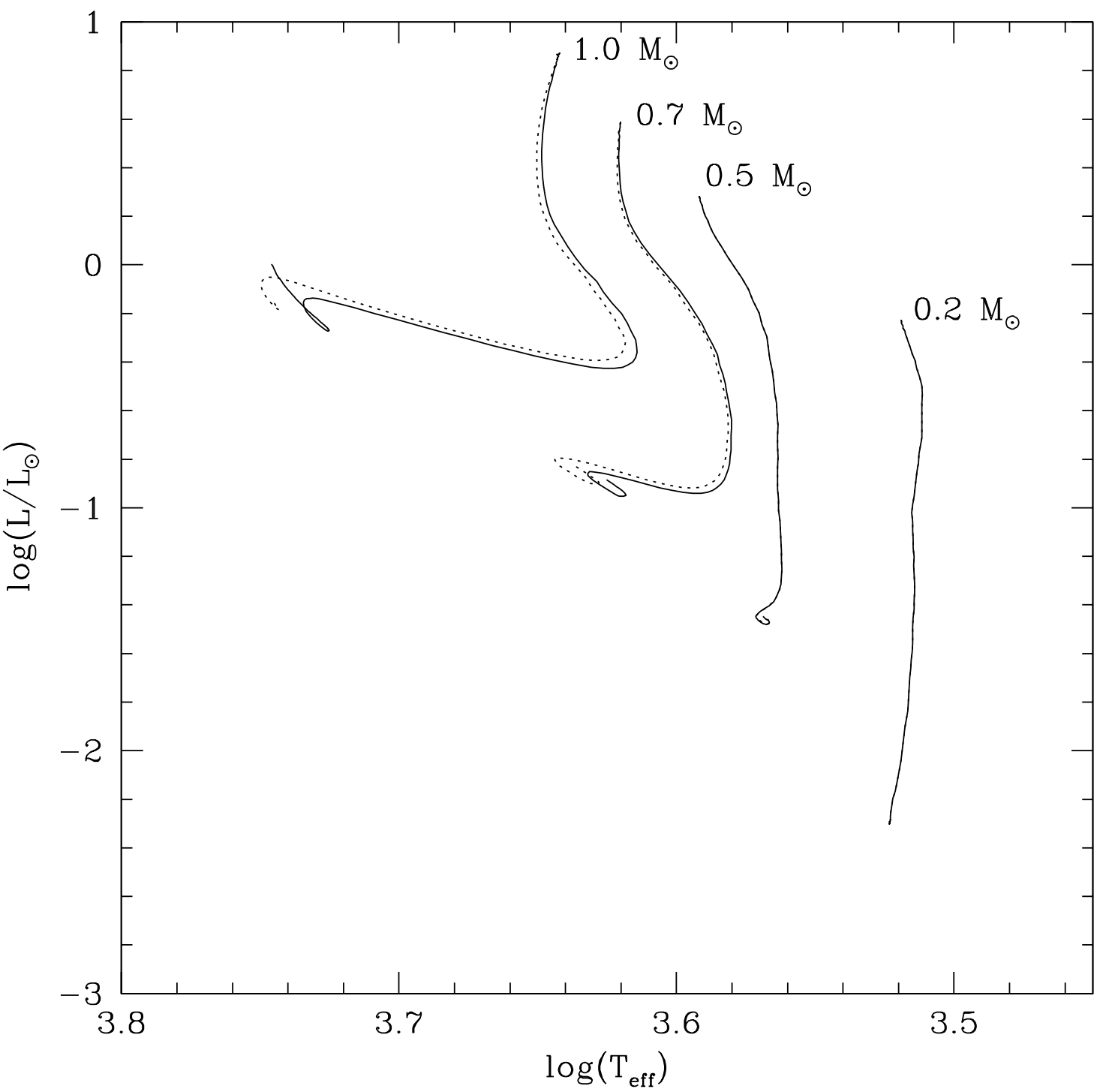

Fig. 2.- Evolutionary tracks for rotating stars under different assumptions about internal angular momentum transport. The solid tracks are stars which have differentially rotating radiative cores and rigidly rotating convection zones, while the dashed lines show the tracks for stars which are constrained to rotate as solid bodies. Stars of the same mass have the same surface rotation rate at the zero age main sequence. Since the low mass stars are fully convective throughout their pre-main sequence lifetime, they always rotate as solid bodies, and so the two tracks are identical. For the higher mass stars, the tracks are the same while the star is on the early pre-main sequence. When the star begins to develop a significant radiative core, however, the two cases have different evolutionary paths. 


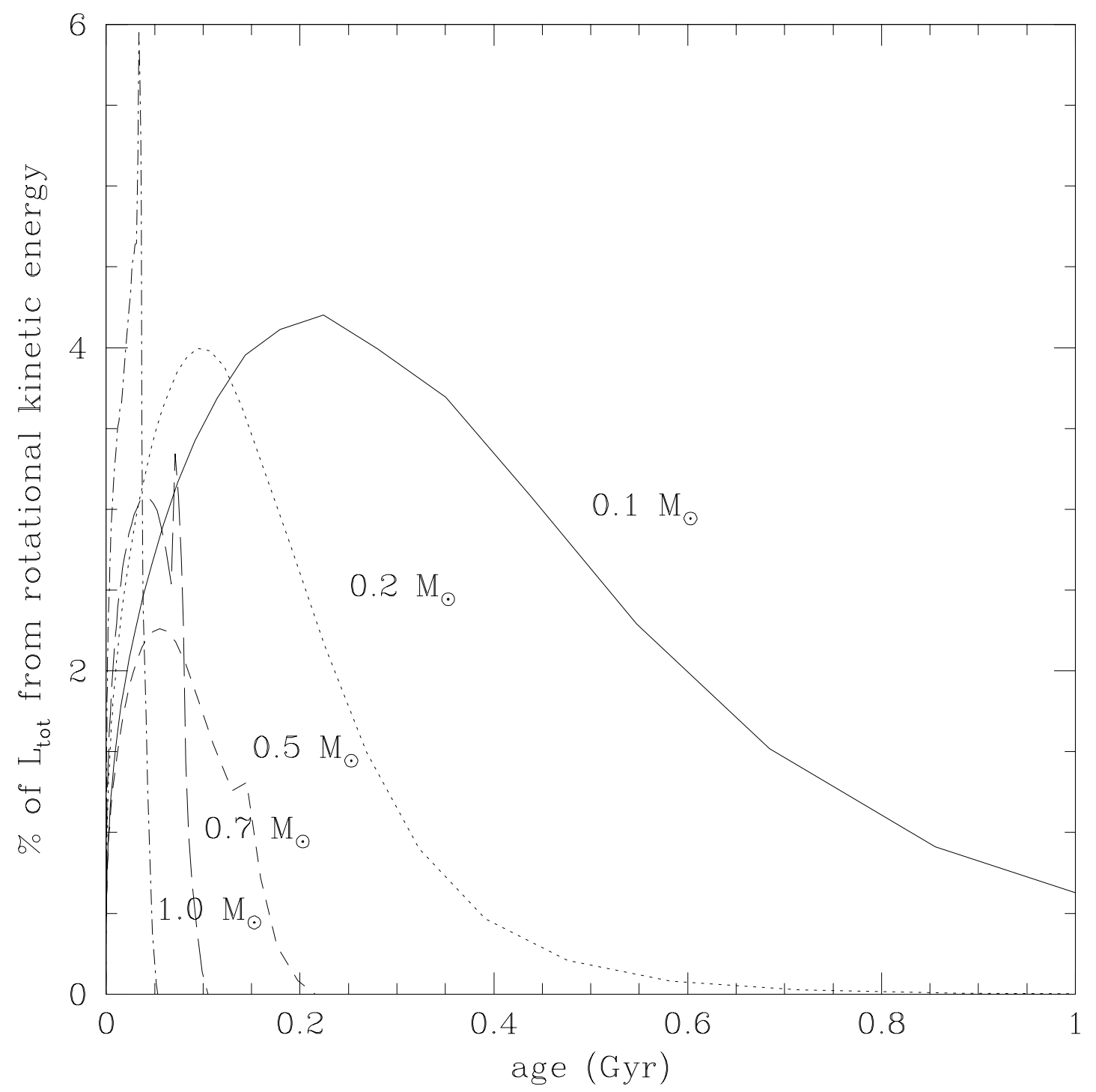

Fig. 3.- The percentage contribution to the total luminosity by the rotational kinetic energy, as a function of age. The maximum contribution is $6 \%$, for the $1.0 \mathrm{M}_{\odot}$ star, but for a short time. For the $0.1 \mathrm{M}_{\odot}$ star, the contribution of rotation kinetic energy lasts the longest time. In either case, the effect on the evolutionary timescale and position in the HR diagram is insignificant. 


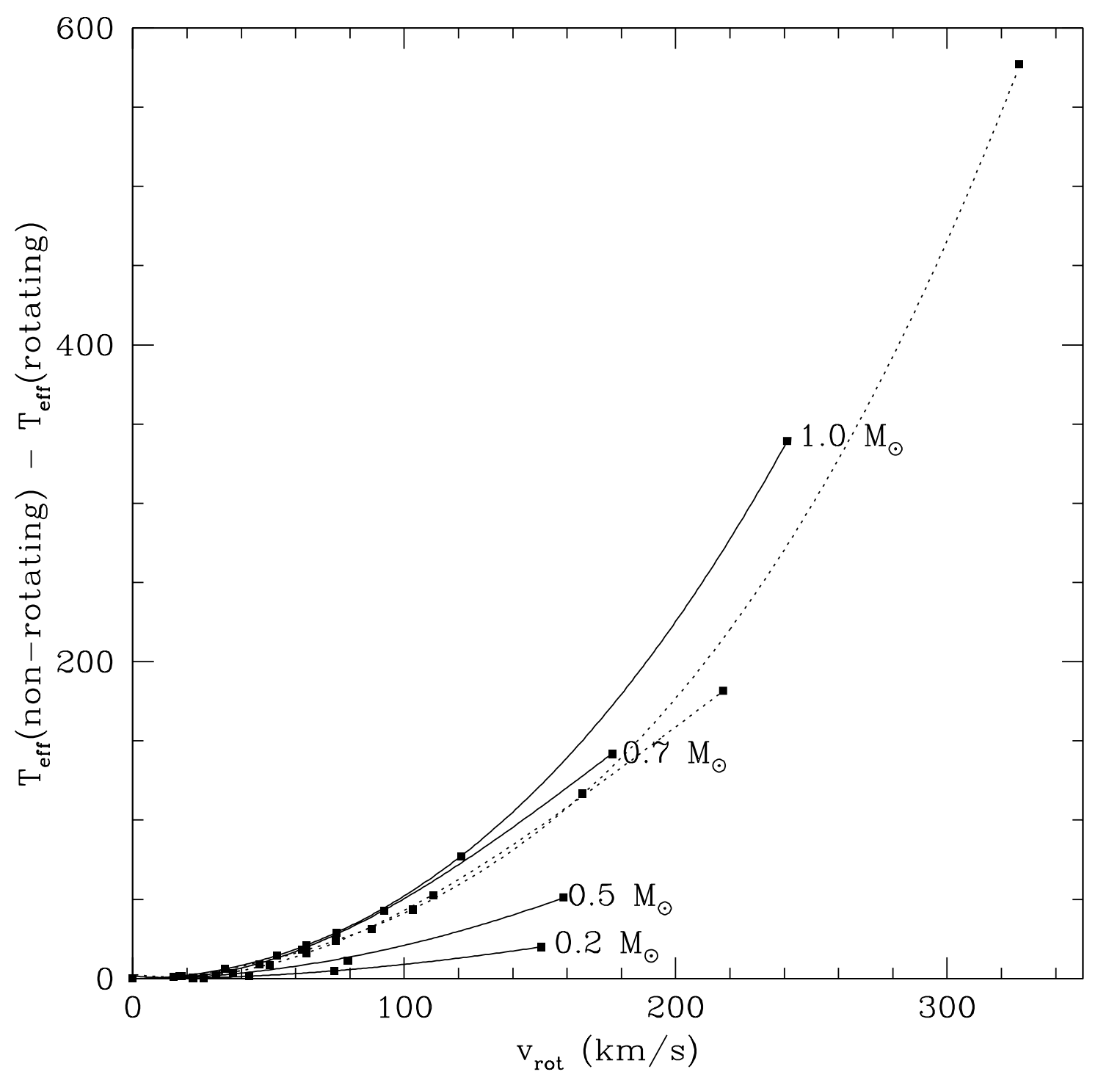

Fig. 4.- Difference between effective temperatures of rotating and non-rotating stars at the same zero age main sequence, as a function of surface rotation velocity. The different lines correspond to stars of different masses, with the highest masses demonstrating the largest difference in temperature. Models which allow for differential rotation are plotted using solid lines, while the solid body rotators are plotted as dashed lines. A difference of a $100 \mathrm{~K}$ or more will significantly affect the mapping of stellar mass on observed effective temperature. 


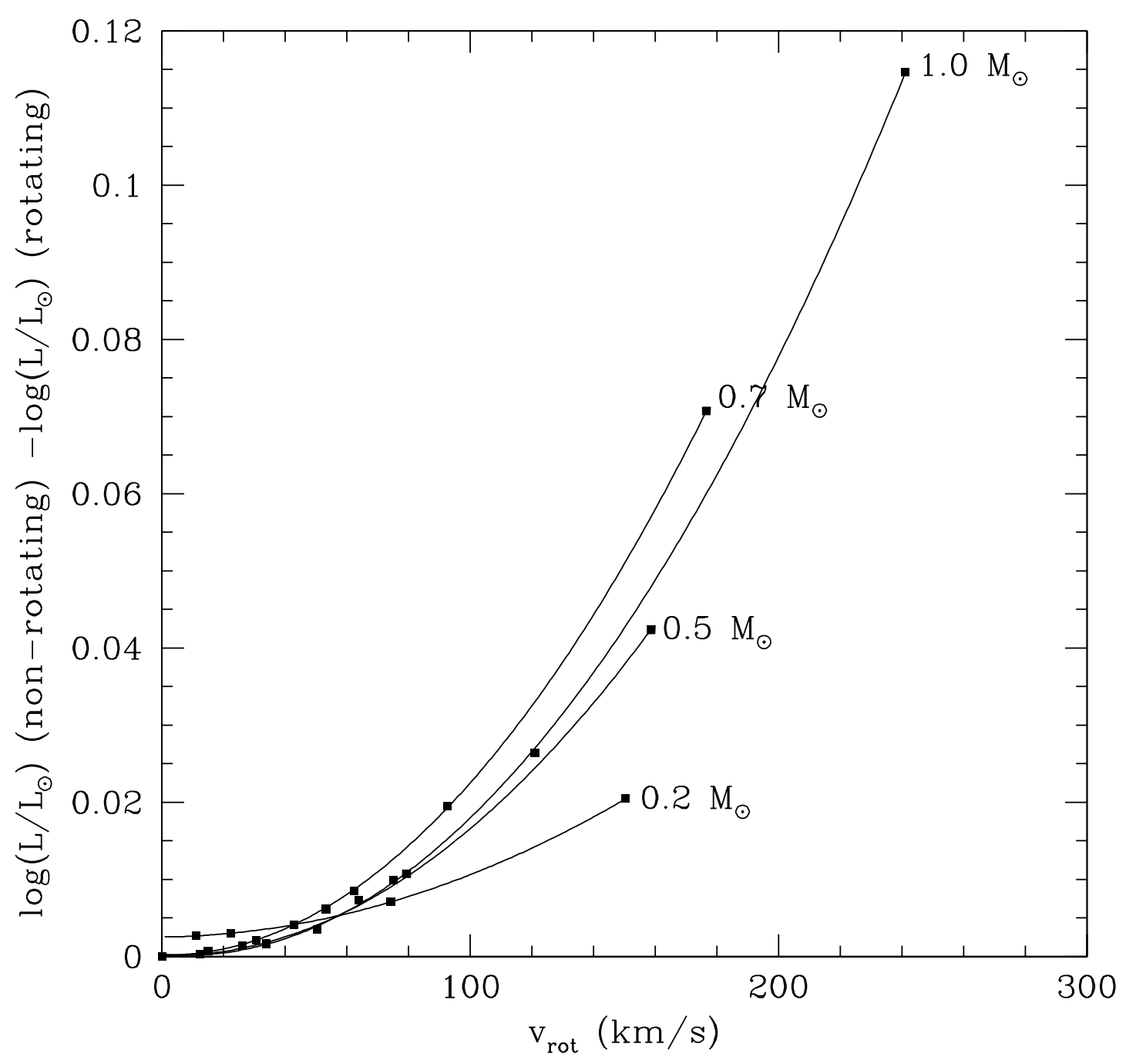

Fig. 5.- Difference between luminosity of rotating and non-rotating stars at the zero age main sequence, as a function of surface rotation velocity. The highest mass stars demonstrate the largest different in luminosity. However, even for $1 \mathrm{M}_{\odot}$, the difference in luminosity is not significant, unlike the difference in effective temperature caused by rotation. 


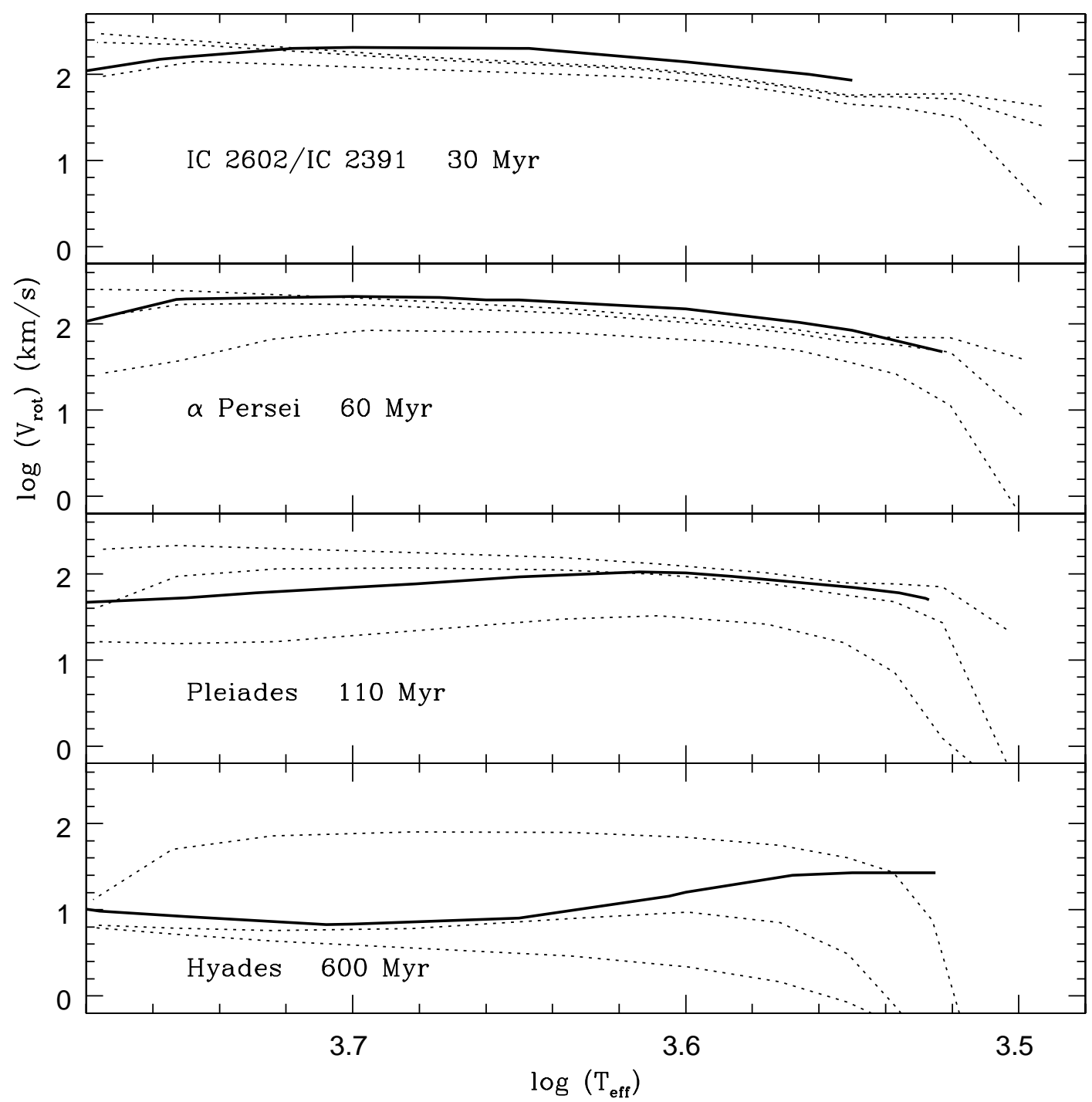

Fig. 6. - Solid body models compared to the upper envelope of the open cluster data at different ages. A mass dependent $\omega_{\text {crit }}$ was used. Three normalizations are presented here. From top to bottom of each frame, we used 5, 10 and $20 \omega_{\odot}$ at $1.0 \mathrm{M}_{\odot}$. The thick solid line represents the upper envelope of the observational data. 


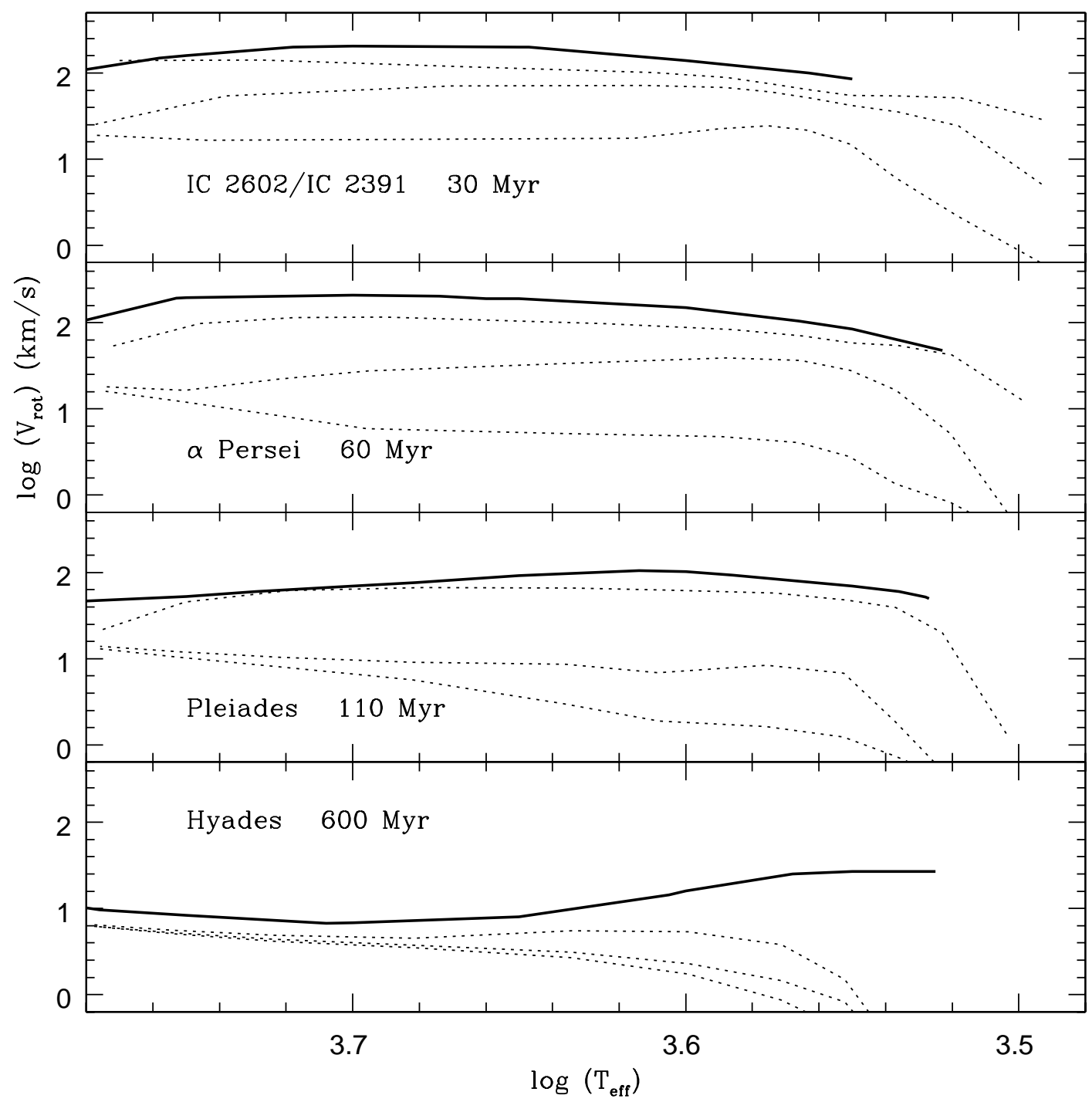

Fig. 7.- As figure 6, with differentially rotating models. Notice the effect of very rapid rotation in the youngest clusters on the temperature of the highest mass models. If stars begin their lives with 4 day periods, the fast rotators in IC 2602 and IC 2391 with temperatures higher than $\log T_{\text {eff }}=3.68$ must have masses higher than $1.1 \mathrm{M}_{\odot}$. 


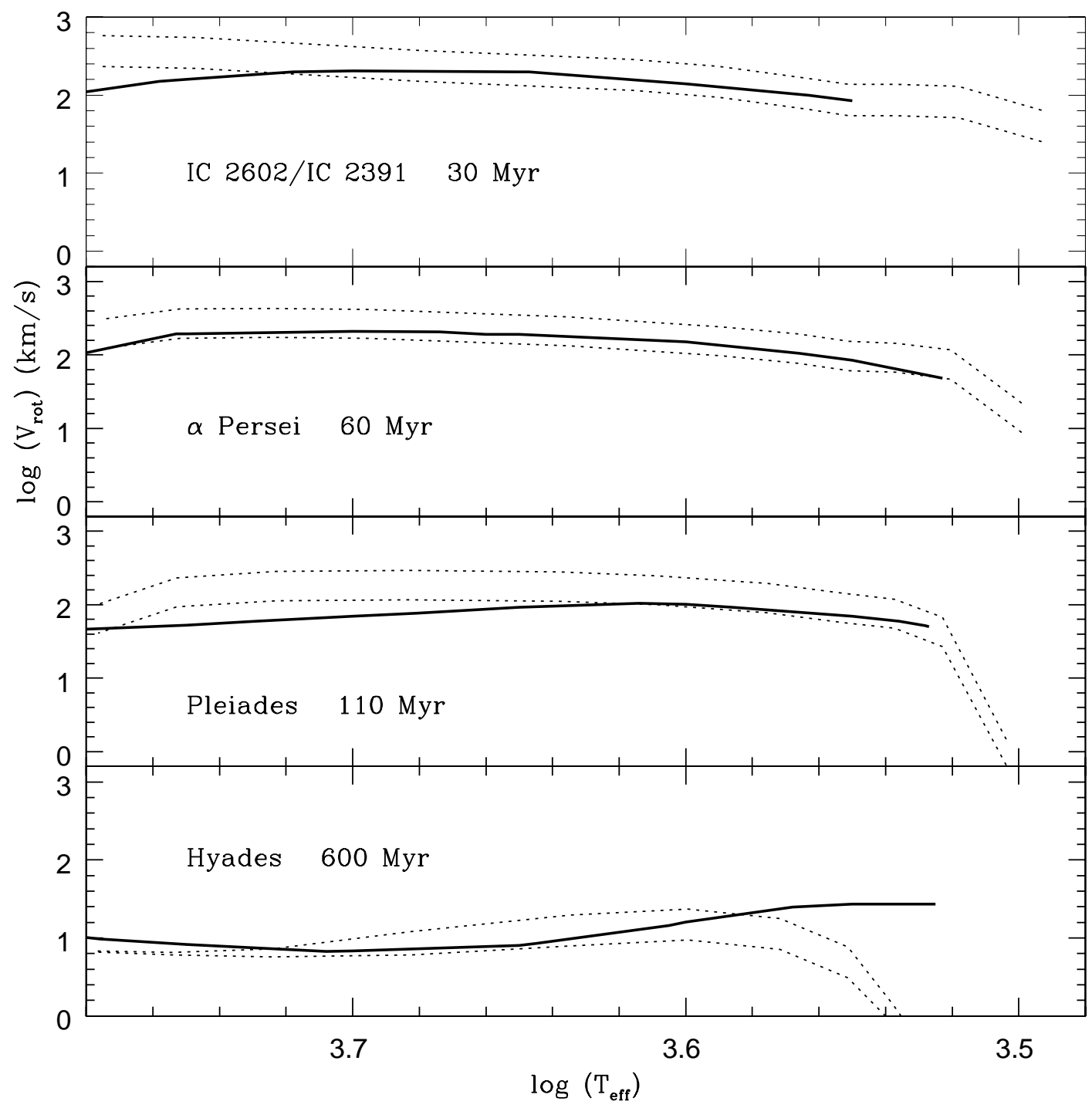

Fig. 8. - Solid body models compared to the upper envelope of the open cluster data at different ages. A mass dependent $\omega_{\text {crit }}$ was used, normalized to $10 \omega_{\odot}$ at $1.0 \mathrm{M}_{\odot}$. The upper line corresponds to models with an initial rotation period of 4 days, and the lower line shows models with an initial rotation period of 10 days. 


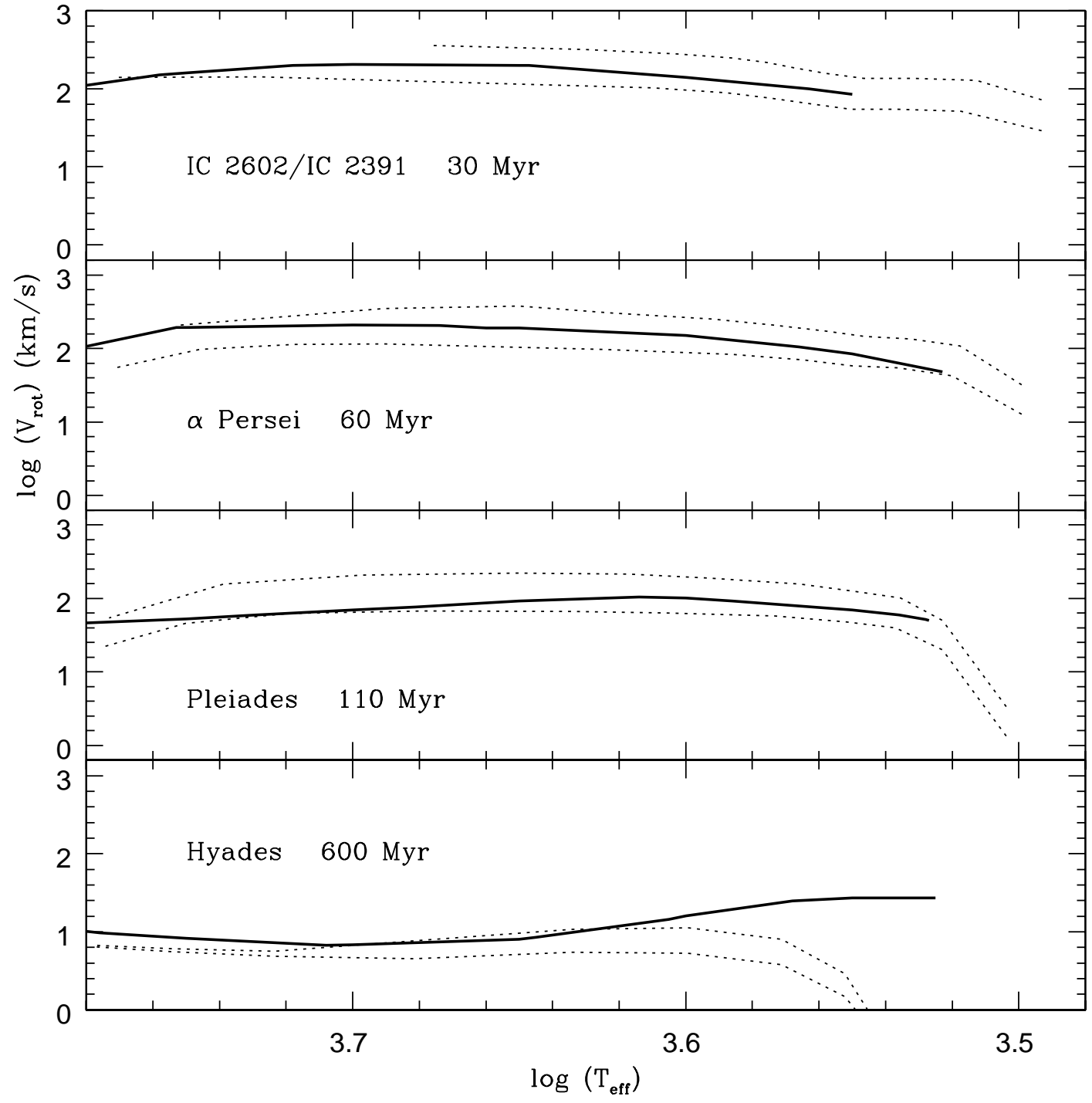

Fig. 9.- As figure 8, with differentially rotating models. The mass dependent $\omega_{\text {crit }}$ was normalized to $5 \omega_{\odot}$ at $1.0 \mathrm{M}_{\odot}$. 


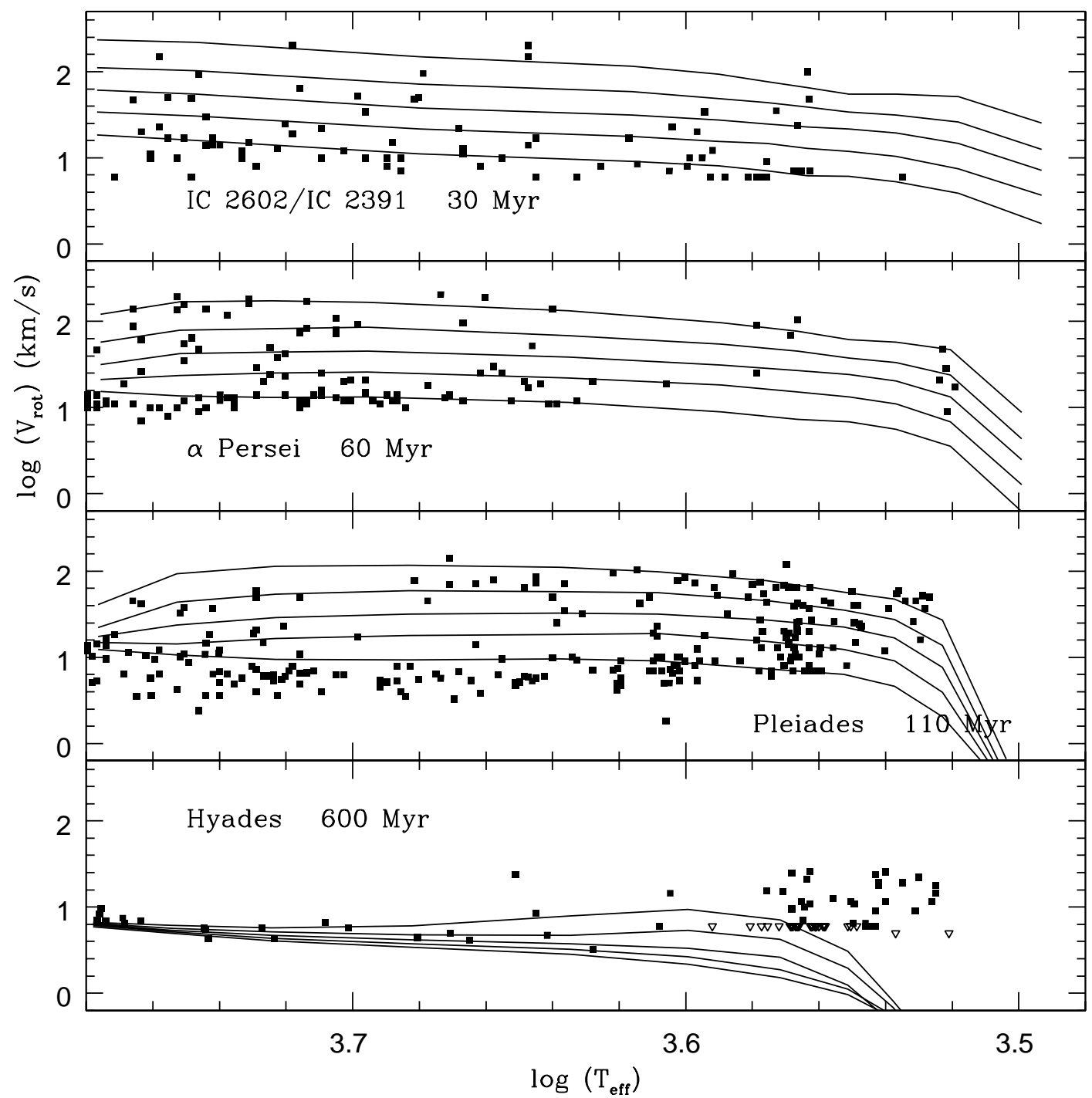

Fig. 10.- Solid body models compared to open cluster data at different ages. A mass dependent $\omega_{\text {crit }}$ was used, normalized to $10 \omega_{\odot}$ at $1.0 \mathrm{M}_{\odot}$. Five different values of the disk locking lifetime were used: 0, 0.3, 1, 3 and 10 Myr (from top to bottom). 


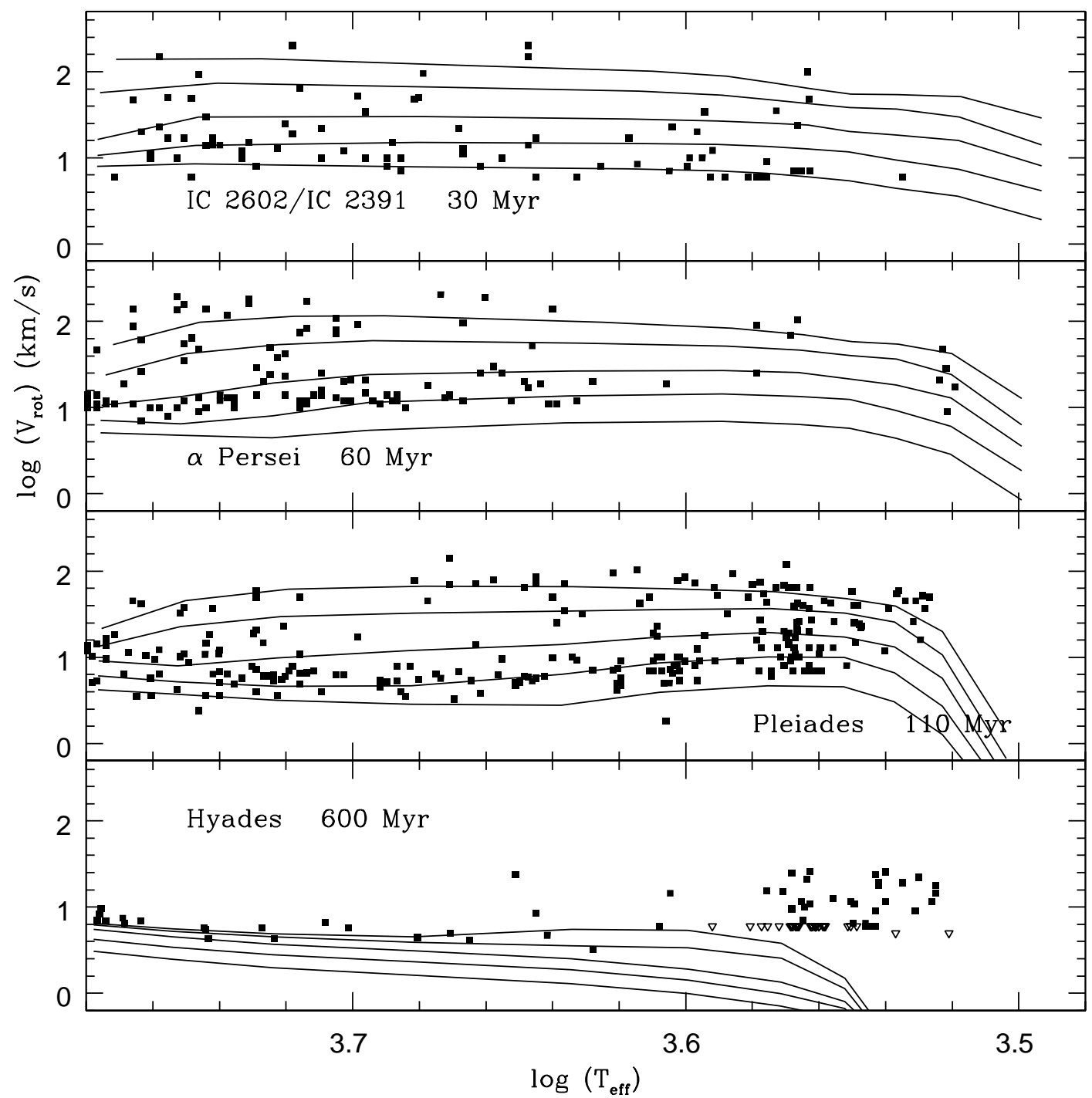

Fig. 11. - Differentially rotating models compared to open cluster data at different ages. The mass dependent $\omega_{\text {crit }}$ was normalized to $5 \omega_{\odot}$ at $1.0 \mathrm{M}_{\odot}$. Five different values of the disk locking lifetime were used: 0, 0.3, 1, 3 and 10 Myr (from top to bottom). 


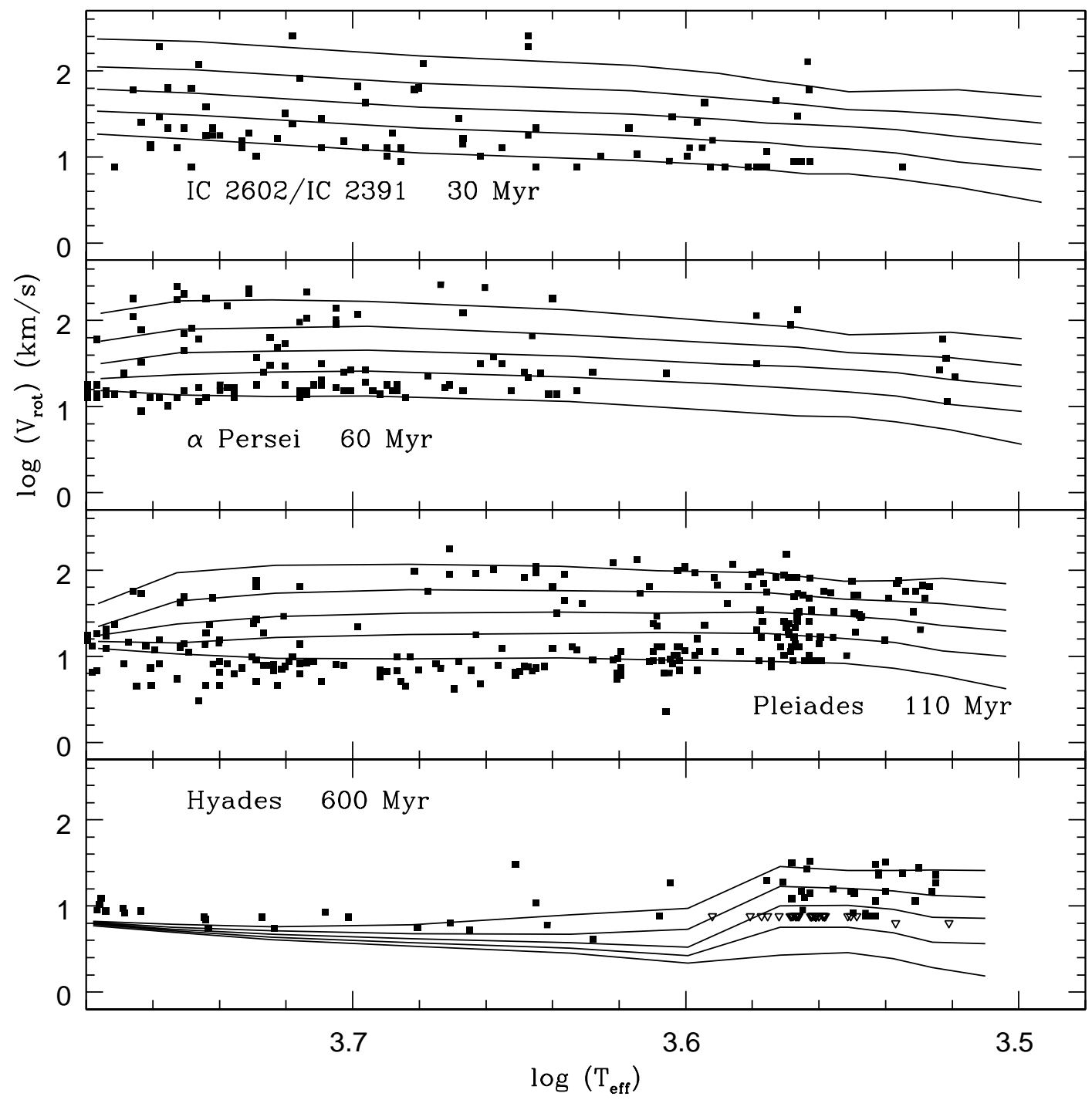

Fig. 12.- Solid body models compared to open cluster data at different ages. Five different values of the disk locking lifetime were used: $0,0.3,1,3$ and $10 \mathrm{Myr}$ (from top to bottom). These models are constrained to more accurately match the Hyades observations below 0.5 $\mathrm{M}_{\odot}$. 


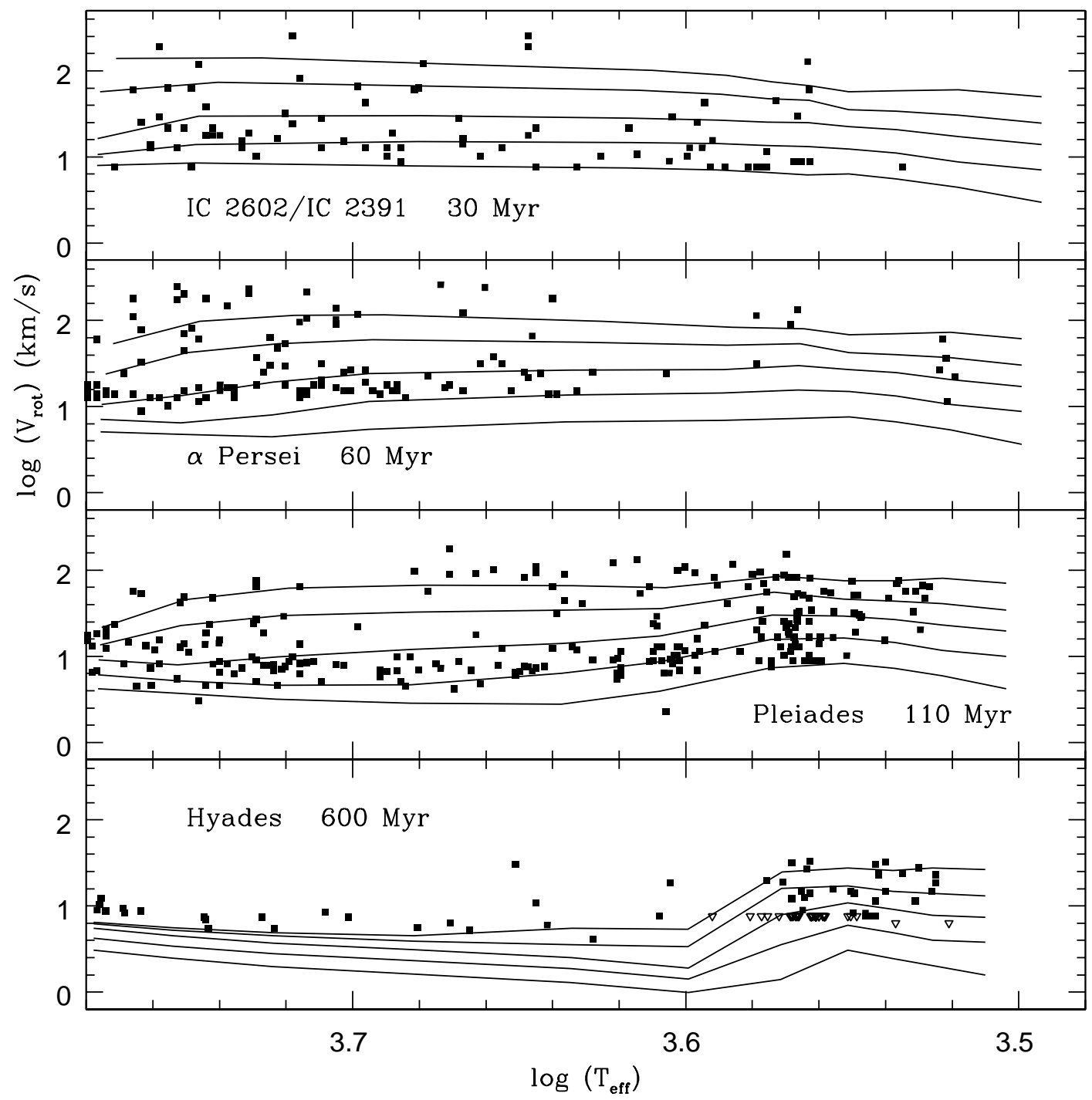

Fig. 13.- Differentially rotating models compared to open cluster data at different ages. Five different values of the disk locking lifetime were used: 0, 0.3, 1, 3 and $10 \mathrm{Myr}$ (from top to bottom). These models are constrained to more accurately match the Hyades observations below $0.5 \mathrm{M}_{\odot}$. 

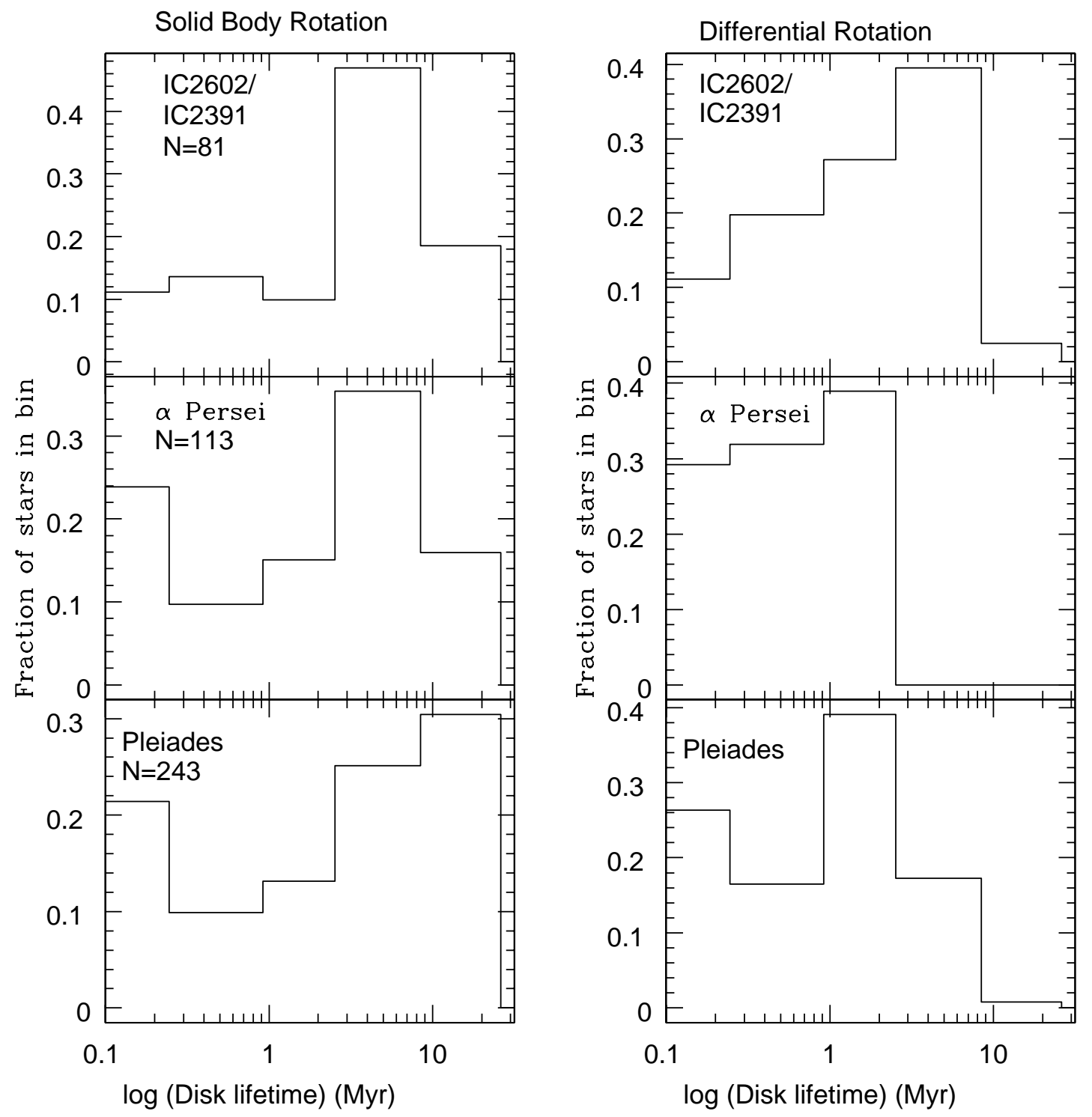

Fig. 14.- Distributions of disk lifetimes for the IC 2602 and IC 2391, $\alpha$ Persei and Pleiades clusters, based on the models presented in figures 12 and 13. The models which constrain the stars to rotate as solid bodies require very long disk lifetimes, in disagreement with observations. 


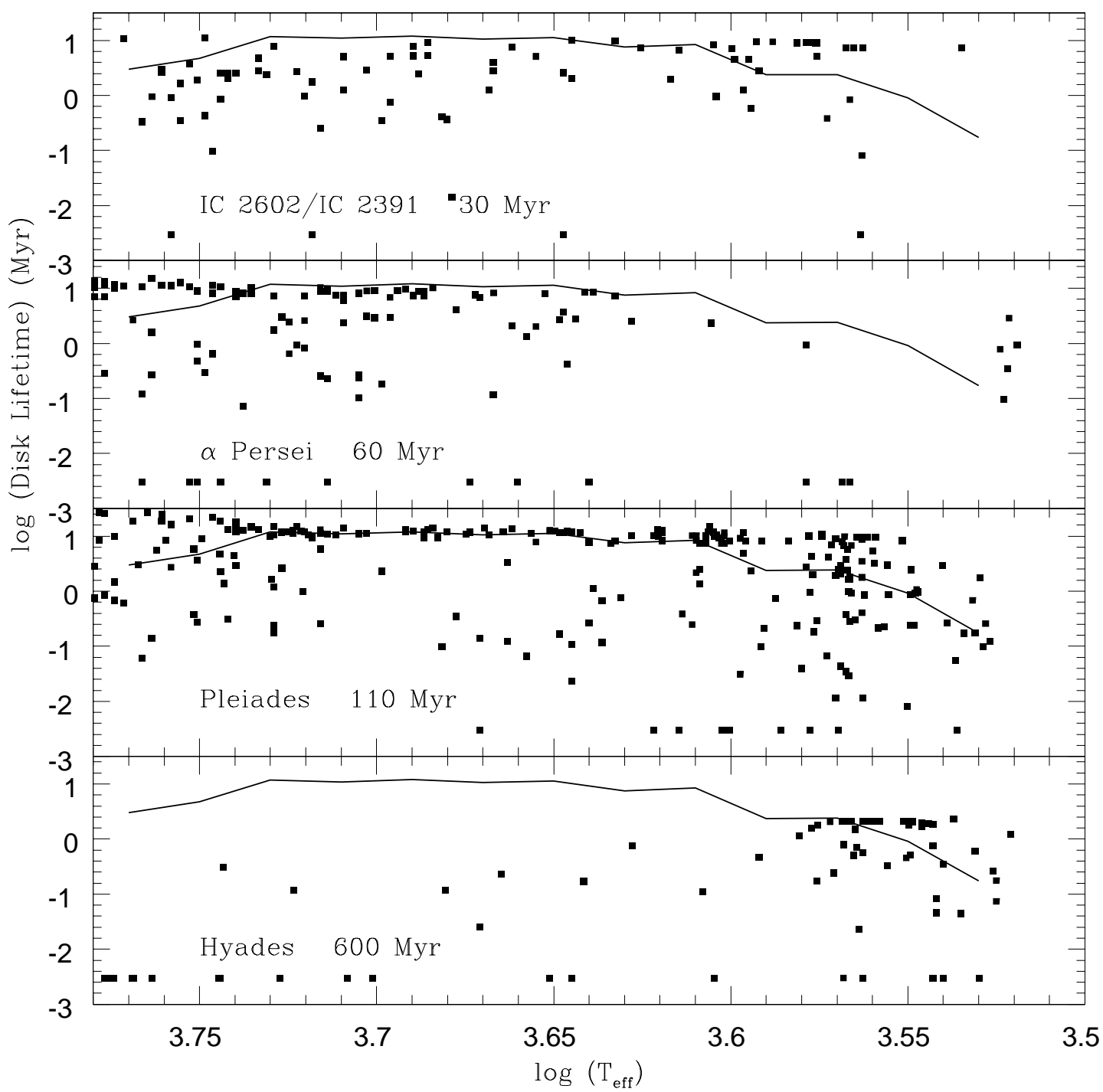

Fig. 15.- Disk lifetimes as a function of effective temperature, based on the solid body models presented in figure 12. The solid line shows the median of the Pleiades disk lifetimes for the models which match the Hyades observations (shown in figure 12). 


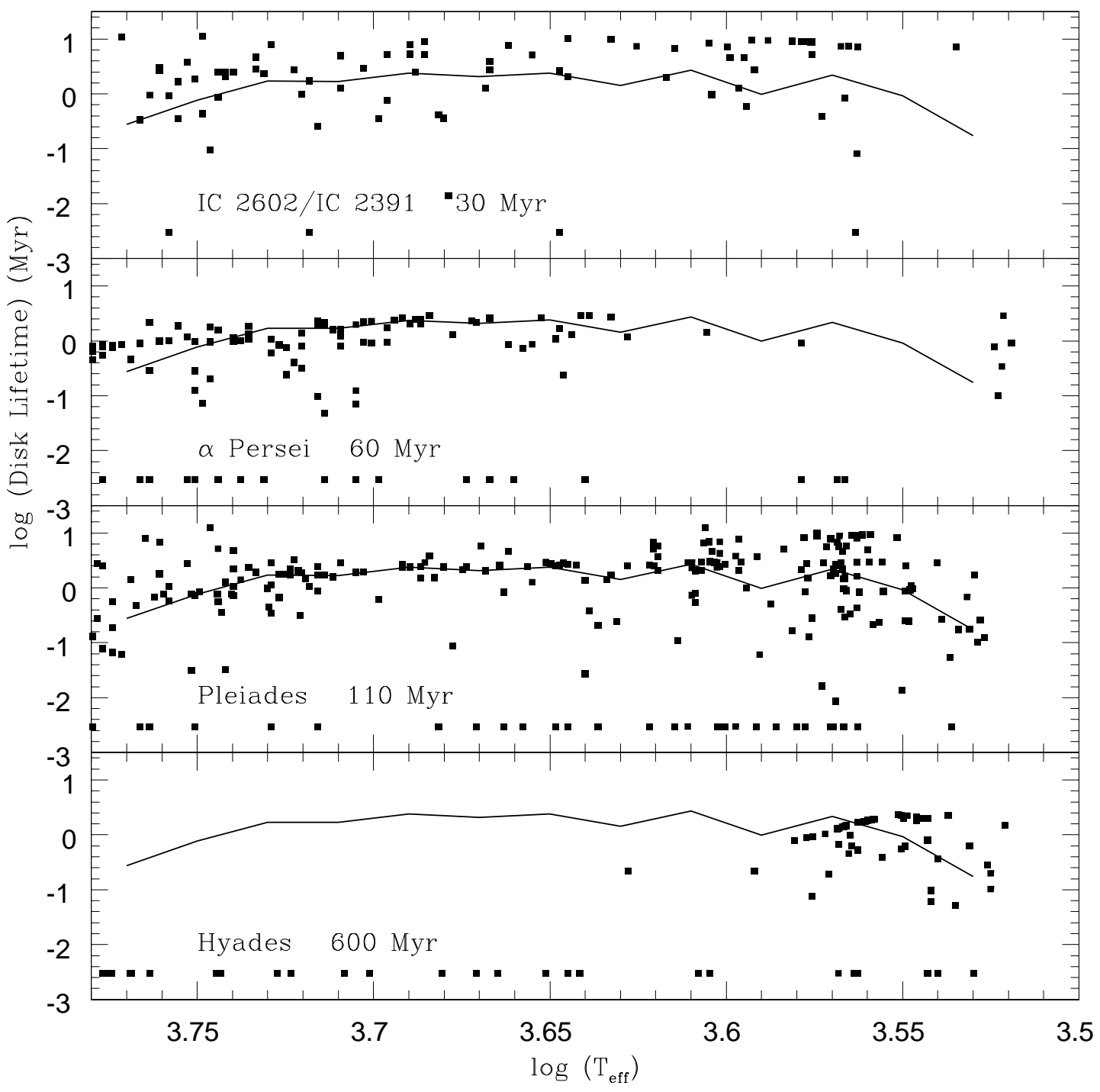

Fig. 16.- As figure 15 with differentially rotating models. The solid line shows the median Pleiades disk lifetimes as derived from the model shown in figure 13. 


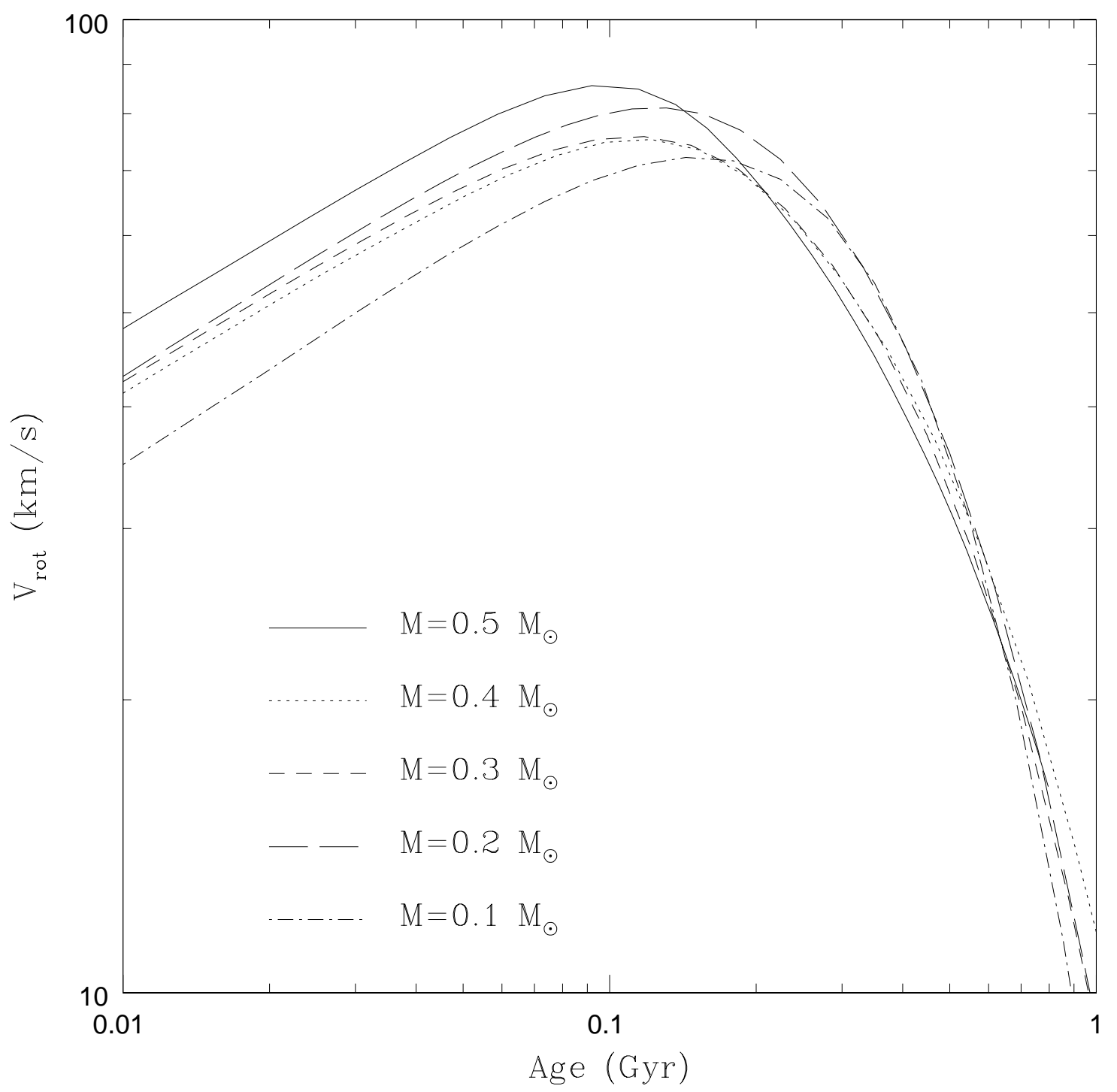

Fig. 17. - Rotation velocity as a function of time for the low mass stars $\left(\mathrm{M} \leq 0.5 \mathrm{M}_{\odot}\right)$ from the preferred models shown in figure 13. The models shown here include no disk locking. 
Table 1. Zero Age Main Sequence Information for Rotating Models with an Initial Period of 8 Days, Differential Rotation and no Angular Momentum Loss

\begin{tabular}{cccc}
\hline \hline Mass $\left(\mathrm{M}_{\odot}\right)$ & $\log T_{\text {eff }}$ & $\log \left(L / L_{\odot}\right)$ & Age $(\mathrm{Myr})$ \\
\hline 0.2 & 3.526 & -2.282 & 890 \\
0.5 & 3.572 & -1.450 & 280 \\
0.7 & 3.634 & -0.881 & 230 \\
1.0 & 3.752 & -0.159 & 27 \\
\hline
\end{tabular}

Table 2. Polynomial Coefficients: $T_{\text {eff }}^{\text {norot }}-T_{\text {eff }}^{r o t}=A v_{\text {rot }}^{3}+B v_{\text {rot }}^{2}+C v_{r o t}+D$

\begin{tabular}{ccccc}
\hline \hline Mass $\left(\mathrm{M}_{\odot}\right)$ & $\mathrm{A}$ & $\mathrm{B}$ & $\mathrm{C}$ & $\mathrm{D}$ \\
\hline 0.2 & $-9.43 \times 10^{-7}$ & $1.08 \times 10^{-3}$ & $-7.71 \times 10^{-3}$ & -0.123 \\
0.5 & 0.0 & $1.87 \times 10^{-3}$ & $2.98 \times 10^{-2}$ & -0.746 \\
0.7 & $-1.49 \times 10^{-5}$ & $8.13 \times 10^{-3}$ & -0.176 & 1.62 \\
1.0 & $5.56 \times 10^{-6}$ & $4.37 \times 10^{-3}$ & $3.19 \times 10^{-2}$ & -0.307 \\
0.7 solid body & $-1.22 \times 10^{-5}$ & $7.43 \times 10^{-3}$ & -0.217 & 2.63 \\
1.0 solid body & $1.02 \times 10^{-5}$ & $1.53 \times 10^{-3}$ & 0.184 & -2.66 \\
\hline
\end{tabular}

